\title{
INTEGRATION BY PARTS ON GENERALIZED MANIFOLDS AND APPLICATIONS ON QUASIREGULAR MAPS
}

\author{
Ville Kirsilä \\ University of Jyväskylä, Department of Mathematics and Statistics \\ P. O. Box 35 (MaD), FI-40014 University of Jyväskylä, Finland; ville.kirsila@jyu.fi
}

\begin{abstract}
We establish an integration by parts formula for $\mathbf{R}^{n}$-valued Sobolev maps on generalized manifolds and study some applications to quasiregular mappings, such as higher integrability of the Jacobian. For quasiregular mappings we also give a modulus of continuity estimate depending on the local index.
\end{abstract}

\section{Introduction}

We study quasiregular mappings from a generalized $n$-manifold to $\mathbf{R}^{n}$. One motivation for our study arises from the difficult question of deciding when a given metric space is locally bi-Lipschitz equivalent to a Euclidean space, or when there exist locally homeomorphic quasiregular maps from the given space to a Euclidean space. For those and related questions, see for instance [4],[5], [7] and [8].

We prove a version of Stokes theorem for Lipschitz and Sobolev maps on generalized manifolds. To be more precise, we prove that if $\mathcal{S}$ is a metrically oriented Ahlfors n-regular generalized manifold (see Section 2 for definitions) and if $f$ is a Lipschitz map from $\mathcal{S}$ to $\mathbf{R}^{n}$ and one of the component functions of $f$ has a compact support on some relatively compact set $\Omega \subset \mathcal{S}$ then the integral of the Jacobian of $f$ over $\Omega$ vanishes. If in addition $\mathcal{S}$ supports a weak $n$-Poincaré inequality, we can use an approximation argument to obtain a version of Stokes theorem for Sobolev maps of class $N_{\text {loc }}^{1, n}\left(\mathcal{S}, \mathbf{R}^{n}\right)$, see Theorem 3.1. We combine methods from analysis, topology and geometric measure theory. In particular, the proof of this result is based on topological degree and coarea formula, and is geometric in nature.

In [8] Heinonen and Sullivan, among other things, proved that if $\mathcal{S} \subset \mathbf{R}^{m}$ is a metrically oriented generalized n-manifold then $\mathcal{S}$ is a local Stokes cycle, i.e. each point $p \in \mathcal{S}$ has a neighborhood $U_{p}$ such that if $\omega$ is a Whitney $(n-1)$-form with support in $U_{p}$ then $\int_{U_{p}} d \omega=0$. A differential form $\omega$ of the ambient space $\mathbf{R}^{m}$ is called a Whitney form if it has distributional exterior derivative $d \omega$ and both $\omega$ and $d \omega$ have bounded measurable coefficients. In Theorem 3.1 it is not assumed that the mapping or it's Jacobian can be extended to the ambient space.

Therorem 3.1 allows us to generalize several well-known results on quasiregular maps to maps $f: \mathcal{S} \rightarrow \mathbf{R}^{n}$, where $\mathcal{S}$ is as above. In particular, we prove higher integrability of the Jacobian and a compactness result for $K$-quasiregular mappings, see Theorem 4.4 and 4.6 respectively. For those and many other classical results on quasiregular maps on Euclidean spaces see for instance [1], [9], [15] and [16]. It

doi:10.5186/aasfm.2016.4123

2010 Mathematics Subject Classification: Primary 30C65, 30L10.

Key words: Generalized manifolds, integration by parts, quasiregular mappings.

The author was financially supported by Finnish National Doctoral Programme in Mathematics and its Applications and by the Academy of Finland, project 257482. 
is worth noticing, that in [10], where the Reshetnyak's theorem was proved in this generality, the definition of quasiregular mappings included the assumption that the map is continuous. However, it follows from higher integrability that this assumption can be removed, see Corollary 4.5.

In the second part of this paper, starting from the analytic definition of quasiregular maps, we prove a local modulus of continuity, see Theorem 7.6. This gives a local improvement to the classical Hölder-continuity estimate, see Corollary 4.5, [16, III. Theorem 1.11] or [10, Theorem 6.3]. We also prove that for a nonconstant quasiregular map, so-called linear inner distortion is bounded by a constant depending only on the data assosiated with domain space and on the distortion constant, see Theorem 7.3. The idea of the proof is similar to one given in [16, Theorem III.4.7] and originally in [12], where the result was proven in Euclidean case. However also new methods are needed. In particular, we give a modulus inequality that acts as a substitute for Väisälä's inequality.

\section{Preliminaries}

Definition 2.1. Let $(X, \mu)$ be a metric measure space, let $\mu$ be Borel regular measure. Moreover, let $\Gamma$ be a collection of paths in $X$, and $p \geq 1$. We say that a Borel function $\rho: X \rightarrow[0, \infty]$ is admissible for $\Gamma, \rho \in \operatorname{Adm} \Gamma$, if

$$
\int_{\gamma} \rho d s \geq 1
$$

for every locally rectifiable $\gamma \in \Gamma$. The $p$-modulus of $\Gamma$, denoted by $\operatorname{Mod}_{p} \Gamma$ is,

$$
\inf \int_{X} \rho(x)^{p} d \mu
$$

where the infimum is taken over all admissible functions $\rho \in \operatorname{Adm} \Gamma$.

If $A, B \subset X$ we write $\Gamma(A, B)$ for the family of all locally rectifiable paths connecting $A$ to $B$ in $X$. If $\Gamma$ is a family of paths and $f$ a continuous map, we write $f \Gamma$ for the path family $\{f \circ \gamma: \gamma \in \Gamma\}$. For the basic properties of the modulus see [6] and [19].

In any metric space $X$ the distance between elements $x$ and $y$ is denoted by $|x-y|$. We write $\alpha_{n}$ for the Lebesgue measure of the unit ball in $\mathbf{R}^{n}$ and $\omega_{n-1}$ for the $(n-1)$-dimensional Hausdorff measure of unit sphere in $\mathbf{R}^{n}$. The $n$-dimensional Hausdorff measure is denoted by $\mathcal{H}^{n}$. If $u: X \rightarrow \mathbf{R}$, we write spt $u$ for the support of $u$. That is spt $u=\overline{\{x \in X: u(x) \neq 0\}}$. If $u: X \rightarrow \mathbf{R}$ is a Lipschitz function with compact support and spt $u \subset \Omega \subset X$ we write $u \in \operatorname{Lip}_{0}(\Omega)$.

Definition 2.2. Let $X$ be metric space and $Y$ a metric space or $\overline{\mathbf{R}}$. Let $f: X \rightarrow$ $Y$. We say that a Borel function $g: X \rightarrow[0, \infty]$ is an upper gradient of $f$ if

$$
|f(x)-f(y)| \leq \int_{\gamma} g d s
$$

for every locally rectifiable path $\gamma:[0,1] \rightarrow X$ with $\gamma(0)=x$ and $\gamma(1)=y$. Furthermore, we say that $g$ is a $p$-weak upper gradient of $f$, if there exists a path family $\Gamma_{0}$, such that $\operatorname{Mod}_{p}\left(\Gamma_{0}\right)=0$ and (1) holds for every $\gamma \notin \Gamma_{0}$. In the case that $Y=\overline{\mathbf{R}}, f(\gamma(0))=\infty$ and $f(\gamma(1))$ is finite, inequality (1) is understood as requirement $\int_{\gamma} g d s=\infty$. If $f(\gamma(0))=f(\gamma(1))=\infty$, inequality (1) is always satisfied. 
Definition 2.3. Let $(X, \mu)$ be a metric measure space and $p \geq 1$. If $u \in L^{p}(X)$ has a $p$-integrable $p$-weak upper gradient, we say that $u \in N^{1, p}(X)$. Let $\left\{u_{i}\right\}_{i=1}^{\infty}$ be a sequence of functions in $N^{1, p}(X)$. We say that it converges to $u \in N^{1, p}(X)$ in the Newtonian sense, or in $N^{1, p}(X)$, if there exist $p$-weak upper gradients $g_{i}$ of $u-u_{i}$, such that

$$
\int_{X}\left|u(x)-u_{i}(x)\right|^{p} d \mu+\int_{X} g_{i}(x)^{p} d \mu \rightarrow 0, \text { as } i \rightarrow \infty .
$$

Assume now that $f$ maps $X$ to $\mathbf{R}^{m}$. In case that every component function $u$ of $f$ belongs to the Newtonian space $N^{1, p}(X)$, we write $f \in N^{1, p}\left(X, \mathbf{R}^{m}\right)$. Convergence in $N^{1, p}\left(X, \mathbf{R}^{m}\right)$ is defined to be convergence of component functions in $N^{1, p}(X)$. Furthermore we say that $f \in N_{\text {loc }}^{1, p}(X)$ if $f$ has a $p$-weak upper gradient $g$, and $f, g \in L^{p}(\Omega)$, for every compact $\Omega \subset X$. The space $N_{\text {loc }}^{1, p}\left(X, \mathbf{R}^{m}\right)$ is defined by the requirement that each component function belongs to $N_{\text {loc }}^{1, p}(X)$. Convergence in $N_{\text {loc }}^{1, p}\left(X, \mathbf{R}^{m}\right)$ means convergence of the component functions in $N^{1, p}(\Omega)$ for every compact $\Omega \subset X$.

Axioms. In this paper, we assume that $\mathcal{S}$ is a locally connected, path connected and proper metric measure space that is a subset of some Euclidean space and satisfies the following axioms.

- $\mathcal{S}$ is an $n$-rectifiable set.

- $\mathcal{S}$ is Ahlfors $n$-regular.

- $\mathcal{S}$ is linearly locally contractible.

- $\mathcal{S}$ is a metrically oriented $n$-dimensional cohomology manifold.

Next we briefly explain these axioms and discuss some consequences that are relevant for this work.

Definition 2.4. Fix $2 \leq n \leq m$ and assume $\mathcal{S} \subset \mathbf{R}^{m}$ is $\mathcal{H}^{n}$-measurable. We say that $\mathcal{S}$ is $n$-rectifiable if there exist Lipschitz maps $\Psi_{i}: \mathbf{R}^{n} \rightarrow \mathbf{R}^{m}$ such that

$$
\mathcal{H}^{n}\left(\mathcal{S} \backslash \bigcup_{i=1}^{\infty} \Psi_{i}\left(\mathbf{R}^{n}\right)\right)=0
$$

Definition 2.5. Let $Q>0$. We say that $\mathcal{S}$ endowed with a Borel measure $\mu$ is Ahlfors $Q$-regular if there exists a constant $C_{A} \geq 1$, such that

$$
\frac{1}{C_{A}} r^{Q} \leq \mu(B(x, r)) \leq C_{A} r^{Q},
$$

for every ball $B(x, r) \subset \mathcal{S}$ such that $r \leq \operatorname{diam}(\mathcal{S})$.

It is well known that if a locally compact metric space $X$ satisfies the Ahlfors $Q$-regularity condition with some Borel measure $\mu$ then $\mu$ is comparable to $Q$ dimensional Hausdorff measure. Thus we always assume that $\mathcal{S}$ is equipped with the $n$-dimensional Hausdorff measure.

Definition 2.6. We say that a metric space $\mathcal{S}$ is linearly locally contractible if there is a constant $C \geq 1$ such that for every compact $K \subset \mathcal{S}$ there exist $r_{K}>0$ such that $B(x, r)$ is contractible in $B(x, C r)$ whenever $r \leq r_{K}$ and $x \in K$. This means that there exists a homotopy $h: B(x, r) \times[0,1] \rightarrow B(x, C r)$ such that $h(y, 0)=\operatorname{id}_{B(x, r)}$ and $h(y, 1)$ is a constant map.

In order to have a theory of topological degree available, we assume that $\mathcal{S}$ is an oriented cohomology $n$-manifold. For this consider compactly supported AlexanderSpanier cohomology groups with integer coefficients. We assume that $\mathcal{S}$ has local 
cohomology groups of degree $n-1$ and higher equivalent to those of an $n$-manifold. Furthermore we assume that $\mathcal{S}$ is orientable and that the orientation is chosen. By these assumptions, it is possible to define the local degree $\mu(y, f, U)$ whenever $f: \mathcal{S} \rightarrow$ $\mathbf{R}^{n}$ is continuous, $U \subset \mathcal{S}$ is a domain and $y \notin f(\partial U)$. Here and in what follows, domain is an open and connected set. For the precise definition of the degree see [7] and [10]. We say that a continuous $f: \mathcal{S} \rightarrow \mathbf{R}^{n}$ is sense-preserving if $\mu(y, f, U) \geq 0$ whenever defined.

Since $\mathcal{S}$ is $n$-rectifiable for $\mathcal{H}^{n}$-almost every $x \in \mathcal{S}$ there exists an approximate tangent plane $T_{x} \mathcal{S}$ which is an $n$-dimensional vector space, see [13, Theorem 15.19]. The collection of all those planes is called tangent bundle $T \mathcal{S}$. By saying that $\mathcal{S}$ is metrically oriented we mean that the orientation of the tangent bundle $T \mathcal{S}$ is chosen in a way that $\mathcal{H}^{n}$-almost every $x \in \mathcal{S}$ has a sufficiently small neighborhood $U$ satisfying $\mu\left(x, \pi_{x}, U\right)=1$. Here $\pi_{x}: \mathcal{S} \rightarrow T_{x} \mathcal{S}$ is the restriction of orthogonal projection from the ambient space $\mathbf{R}^{m}$ to $T_{x} \mathcal{S}$. For a detailed discussion on metric orientation we refer to [7] and [10]. From now on, we assume that $\mathcal{S}$ is metrically oriented and in particular an orthonormal basis for each tangent plane is chosen. This assumption does not cause any new restrictions. In fact, it is proven in $[8$, Example 3.10] that under our axioms such an orientation for the tangent bundle $T \mathcal{S}$ can be found.

From the definition and basic properties of Alexander-Spanier cohomology it is not too difficult to show that the topological degree satisfies the following properties.

Lemma 2.7. Let $U \subset \mathcal{S}$ be a domain. For every continuous map $f: \mathcal{S} \rightarrow \mathbf{R}^{n}$ the topological degree satisfies the following:

(1) If $\left\{U_{1}, U_{2}, \ldots, U_{k}\right\}$ is a collection of domains with $U_{i} \cap U_{j}=\emptyset$, for every $i \neq j$ and $U \cap f^{-1}(y) \subset \bigcup_{i=1}^{k} U_{i} \subset U$, then

$$
\mu(y, f, U)=\sum_{i=1}^{k} \mu\left(y, f, U_{i}\right)
$$

for every $y \in f(U) \backslash f(\partial U)$.

(2) $\mu(y, f, U)$ is constant on each connected component of $\mathbf{R}^{n} \backslash f(\partial U)$. In particular, $\mu(y, f, U)=0$ for each $y \in f(U)^{c} \backslash f(\partial U)$.

(3) Let $f$ and $g$ be continuous mappings from $\mathcal{S}$ to $\mathbf{R}^{n}$. Let $y \in \mathbf{R}^{n}$ such that there is homotopy $h_{t}$ between $f$ and $g$ with $y \notin h_{t}(\partial U)$ for every $t$. Then $\mu(y, f, U)=\mu(y, g, U)$.

(4) Let $T: \mathbf{R}^{n} \rightarrow \mathbf{R}^{n}$ be a linear bijection. Then $\mu(y, T, U)=\operatorname{sgn}(\operatorname{det} T)$, for every $y \in T(U)$.

It follows from a deep result of Semmes [17] that if a metric measure space $X \subset \mathbf{R}^{m}$ is Ahlfors $n$-regular, has cohomology modules as described above and is also linearly locally contractible, then $X$ supports a 1-Poincaré inequality and therefore by [6, Theorem 7.3.2] and [6, Corollary 8.1.36] the space $\mathcal{S}$ supports a Sobolev-Poincaré inequality. That is, for $p \geq 1$ there exist constants $C_{p} \geq 1$ and $\lambda \geq 1$ such that for every $f \in N_{\text {loc }}^{1, p}(X)$ we have

$$
\left(f_{B}\left|f(x)-f_{B}\right|^{p^{*}} d \mu\right)^{\frac{1}{p^{*}}} \leq C_{p} \operatorname{diam}(B)\left(f_{\lambda B} g(x)^{p} d \mu\right)^{\frac{1}{p}},
$$

whenever $\mathrm{g}$ is a $p$-weak upper gradient of $f$, and ball $B \subset X$ satisfies $\operatorname{diam} B \leq$ $\operatorname{diam} X$. Here $p^{*}=\frac{p n}{n-p}$ and $f_{B}$ is the integral average $f_{B}=f_{B} f=\frac{1}{\mu(B)} \int_{B} f(x) d \bar{\mu}$. 
One consequence of this inequality is that Lipschitz mappings form a dense subset of $N_{\text {loc }}^{1, n}\left(\mathcal{S}, \mathbf{R}^{k}\right)$, see for instance [6, Theorem 7.2.1]. In some of our results including Thoeorem 3.1 and Theorems 4.4 and 4.6 the assumption of linear local contractibility could be removed if one instead assumes that $\mathcal{S}$ is metrically orientable and supports a weak $n$-Poincaré inequality. In fact, Theorem 3.1 holds for Lipschitz mappings even without assumption of the Poincare inequality. However we assume that $\mathcal{S}$ is linearly locally contractible because that assumption is geometrically more clear and implies that $\mathcal{S}$ is metrically orientable and supports a weak Poincaré inequality.

\section{An integration by parts formula}

In this section we prove the following integration by parts formula, which is one of the main results of this paper.

Theorem 3.1. Let $f \in N_{\text {loc }}^{1, n}\left(\mathcal{S}, \mathbf{R}^{n}\right)$. Assume that $\Omega \subset \mathcal{S}$ is a relatively compact open set and $\eta: \mathcal{S} \rightarrow \mathbf{R}$ a Lipschitz map with spt $\eta \subset \Omega$. Then for each $1 \leq k \leq n$,

$$
\int_{\Omega} \eta J_{f} d \mathcal{H}^{n}=-\int_{\Omega} f_{k} J\left(f_{1}, \ldots, f_{k-1}, \eta, f_{k+1}, \ldots, f_{n}\right) d \mathcal{H}^{n} .
$$

Here the Jacobian $J_{f}$ is defined as follows.

Definition 3.2. If $f \in N_{\text {loc }}^{1, n}\left(\mathcal{S}, \mathbf{R}^{k}\right)$ then $f$ is approximately differentiable $\mathcal{H}^{n}$ almost everywhere, see [10, Corollary 2.17]. The approximate derivative is a linear map from the shifted approximate tangent plane, $\operatorname{ap} D f(x): T_{x} \mathcal{S}-x \rightarrow \mathbf{R}^{k}$, see $[2$, Definition 3.2.16] or [10] for the definition. The Jacobian determinant is

$$
J_{f}(x)= \begin{cases}\operatorname{det} \operatorname{ap} D f(x) & \text { if } k=n, \\ \left.\sqrt{\operatorname{det}\left(\operatorname{ap} D f(x) \operatorname{ap} D f(x)^{T}\right.}\right) & \text { if } k<n, \\ \sqrt{\operatorname{det}\left(\operatorname{ap} D f(x)^{T} \operatorname{ap} D f(x)\right)} & \text { if } k>n .\end{cases}
$$

Recall that $\mathcal{S}$ is metrically oriented and thus an orthonormal basis for each tangent plane is chosen. Often, in the case that $f: \mathcal{S} \rightarrow \mathbf{R}^{n}, f=\left(f_{1}, f_{2}, \ldots, f_{n}\right)$ is approximately differentiable $\mathcal{H}^{n}$-almost everywhere, we use notation $J_{f}=J\left(f_{1}, f_{2}, \ldots, f_{n}\right)$. If $f \in N_{\text {loc }}^{1, n}\left(\mathcal{S}, \mathbf{R}^{n}\right)$, the norm of the approximate differential satisfies

$$
\|\operatorname{ap} D f(x)\|=\sup _{|y|=1}|\operatorname{ap} D f(x) y| \leq C(\mathcal{S}) g(x),
$$

for $\mathcal{H}^{n}$-almost every $x \in \mathcal{S}$. Here $g$ can be any $n$-weak, $n$-integrable upper gradient of $f$. If, in addition $f$ is continuous, then $\|\operatorname{ap} D f(\cdot)\|$ is an $n$-weak upper gradient of $f$. For those facts see [10, Lemma 2.28] and [10, Lemma 2.29].

The following coarea formula is stated in greater generality in [2, Theorem 3.2.22].

Theorem 3.3. Let $k \leq n$ and assume $f: \mathcal{S} \rightarrow \mathbf{R}^{k}$ is Lipschitz. Then for every integrable function $g: \mathcal{S} \rightarrow \mathbf{R}$,

$$
\int_{\mathcal{S}} g(x)\left|J_{f}(x)\right| d \mathcal{H}^{n}(x)=\int_{\mathbf{R}^{k}} \int_{f^{-1}(y)} g(x) d \mathcal{H}^{n-k}(x) d \mathcal{H}^{k}(y) .
$$

Besides approximate differentiability there is also a stronger concept of differentiability that turns out to be useful in the proof of Theorem 3.1.

Definition 3.4. We say that $f: \mathcal{S} \rightarrow \mathbf{R}^{k}$ is differentiable at $x \in \mathcal{S}$, if $T_{x} \mathcal{S}$ exists and if there is a map $g: \mathbf{R}^{m} \rightarrow \mathbf{R}^{k}$, which is differentiable at $x$, and coincides with $f$ 
on $\mathcal{S}$. The restriction of $D g(x)$ to the shifted approximate tangent plane $T_{x} \mathcal{S}-x$ is called differential of $f$ at $x$ and denoted by $D f(x)$. That is,

$$
D f(x): T_{x} \mathcal{S}-x \rightarrow \mathbf{R}^{k}, \quad D f(x)=D g(x)_{\mid T_{x} \mathcal{S}-x} .
$$

It is proven in [10, Lemma 2.20] that Lipschitz mappings from $\mathcal{S}$ to a Euclidean space are differentiable $\mathcal{H}^{n}$-almost everywhere. If $f$ is differentiable at $x \in \mathcal{S}$ then it is also approximately differentiable and the differentials $\operatorname{ap} D f(x)$ and $D f(x)$ coincide.

Lemma 3.5. Let $f: \mathcal{S} \rightarrow \mathbf{R}^{n}$ be a Lipschitz map and $\Omega \subset \mathcal{S}$ a relatively compact domain. Then

$$
\mu(y, f, \Omega)=\sum_{x \in f^{-1}(y) \cap \Omega} \operatorname{sgn} J_{f}(x)
$$

for $\mathcal{H}^{n}$-almost every $y \in \mathbf{R}^{n} \backslash f(\partial \Omega)$.

Proof. If $y \in f(\Omega)^{c} \backslash f(\partial \Omega)$, Lemma 2.7(2) gives $\mu(y, f, \Omega)=0$ and the claim is clear. Let $y \in f(\Omega) \backslash f(\partial \Omega)$. By the coarea formula of Theorem 3.3 we may assume that $f^{-1}(y) \cap \Omega$ is finite and $J_{f}(x) \neq 0$ for every $x \in f^{-1}(y) \cap \Omega$. Furthermore, since Lipschitz mappings are differentiable $\mathcal{H}^{n}$-almost everywhere, we may assume that $f$ is differentiable on $f^{-1}(y) \cap \Omega$. For each $x_{i} \in f^{-1}(y) \cap \Omega$ we find a relatively compact domain $U_{i}$ containing $x_{i}$. We may assume that the sets $U_{i}$ are pairwise disjoint. We will show that for every $U_{i}$,

$$
\mu\left(y, f, U_{i}\right)=\operatorname{sgn} J_{f}\left(x_{i}\right) .
$$

Fix $x_{i} \in f^{-1}(y) \cap \Omega$. After translating we may assume that $x_{i}=0$ and $f\left(x_{i}\right)=y=0$. Furthermore, since $\mathcal{S}$ is metrically oriented we may assume that $\mu\left(0, \pi_{0}, U_{i}\right)=1$. Recall that $\pi_{0}: \mathcal{S} \rightarrow T_{0} \mathcal{S}$ is the restriction of orthogonal projection. On the other hand, since $J_{f}(0) \neq 0$ it follows from Lemma 2.7(4) that $\mu\left(0, D f(0), U_{i}\right)=\operatorname{sgn} J_{f}(0)$. Therefore

$$
\mu\left(y, D f(0) \pi_{0}, U_{i}\right)=\operatorname{sgn} J_{f}(0) .
$$

Because $f$ is differentiable at 0 and $J_{f}(0) \neq 0$ we have

$$
0<\lambda:=\inf _{|z|=1}|D f(0) z| \text {. }
$$

In addition, $2\left|\pi_{0} z\right| \geq|z|$, for $z \in \mathcal{S}$ with sufficiently small $|z|$, see [10, Corollary 2.13]. Therefore $\left|D f(0) \pi_{0} z\right| \geq \frac{\lambda}{2}|z|$, whenever $z \in \mathcal{S}$ with sufficiently small $|z|$. By this fact, using the definition of differentiability it is not too difficult to see that $U_{i}$ can be chosen such that, for the set $U_{i}$

$$
g_{t}(z)=t D f(0) \pi_{0} z+(1-t) f(z)
$$

is a homotopy between $f$ and $D f(0) \pi_{0}$ as in Lemma 2.7(3). This gives

$$
\mu\left(0, f, U_{i}\right)=\mu\left(0, D f(0) \pi_{0}, U_{i}\right)=\operatorname{sgn} J_{f}(0),
$$

and proves (5). Now the claim follows from Lemma 2.7(1).

The following lemma based on a simple geometric observation is a key towards Theorem 3.1.

Lemma 3.6. Let $f: \mathcal{S} \rightarrow \mathbf{R}^{n}$ be a Lipschitz map and let $\Omega \subset \mathcal{S}$ be a relatively compact open set. Assume that for some $1 \leq k \leq n$ the $k$ :th component function $f_{k}$ has compact support on $\Omega$. Then

$$
\int_{\Omega} J_{f}(x) d \mathcal{H}^{n}(x)=0
$$


Proof. Assume first that $\Omega \subset \mathcal{S}$ is connected. Since spt $f_{k} \subset \Omega, f$ maps $\partial \Omega$ into the hyperplane $\left\{y \in \mathbf{R}^{n}: y_{k}=0\right\}$ and thus $\mathcal{H}^{n}(f(\partial \Omega))=0$. By the coarea formula of Theorem 3.3 and by Lemma 3.5 we have

$$
\begin{aligned}
\int_{\Omega} J_{f}(x) d \mathcal{H}^{n}(x) & =\int_{\Omega}\left|J_{f}(x)\right| \operatorname{sgn} J_{f}(x) d \mathcal{H}^{n}(x) \\
& =\int_{f(\Omega)} \sum_{x \in f^{-1}(y) \cap \Omega} \operatorname{sgn} J_{f}(x) d \mathcal{H}^{n}(x)=\int_{f(\Omega)} \mu(y, f, \Omega) d \mathcal{H}^{n}(y) .
\end{aligned}
$$

Thus it suffices to show that $\mu(y, f, \Omega)=0$ for $\mathcal{H}^{n}$-almost every $y \in \mathbf{R}^{n} \backslash f(\partial \Omega)$. Since $f(\bar{\Omega}) \subset \mathbf{R}^{n}$ is compact we find $y^{\prime} \in\left\{y \in \mathbf{R}^{n}: y_{k}>0\right\} \backslash f(\bar{\Omega})$. In particular $y^{\prime}$ satisfies $\mu\left(y^{\prime}, f, \Omega\right)=0$. Since $f(\partial \Omega) \subset\left\{y \in \mathbf{R}^{n}: y_{k}=0\right\}$ we infer that each point of $\left\{y \in \mathbf{R}^{n}: y_{k}>0\right\}$ lies in the same connected component of $\mathbf{R}^{n} \backslash f(\partial \Omega)$ with $y^{\prime}$ and therefore by Lemma $2.7(2) \mu(y, f, \Omega)=0$ for every $y \in\left\{y \in \mathbf{R}^{n}: y_{k}>0\right\}$. A similar reasoning shows that $\mu(y, f, \Omega)=0$ for every $y \in\left\{y \in \mathbf{R}^{n}: y_{k}<0\right\}$. This proves the claim in the case that $\Omega$ is connected. Now consider the case that $\Omega$ is not connected but open and relatively compact. In this case $\Omega$ consists of countably many connected components $\left\{\Omega_{i}\right\}_{i}$ and each of them is a relatively compact. Since $\operatorname{spt} f_{k} \subset \Omega$ we have $f_{k}\left(\partial \Omega_{i}\right)=0$ and therefore $\int_{\Omega_{i}} J_{f} d \mathcal{H}^{n}=0$ as above. Thus

$$
\int_{\Omega} J_{f} d \mathcal{H}^{n}(x)=\sum_{i} \int_{\Omega_{i}} J_{f} d \mathcal{H}^{n}(x)=0 .
$$

This completes the proof.

The following corollary gives the equality of Theorem 3.1 in the case that $f$ is Lipschitz.

Corollary 3.7. Let $f: \mathcal{S} \rightarrow \mathbf{R}^{n}$ be a Lipschitz map and $\Omega \subset \mathcal{S}$ a relatively compact open set. Let $\eta: \mathcal{S} \rightarrow \mathbf{R}$ be a Lipschitz function with spt $\eta \subset \Omega$. Then equation (3) holds.

Proof. Since our notion of differential satisfy the usual Leibniz rule and because determinant is multilinear we may apply Lemma 3.6 to map

$$
\left(f_{1}, \ldots, f_{k-1}, \eta f_{k}, f_{k+1}, \ldots, f_{n}\right)
$$

and find equality (3) for Lipschitz mappings.

Next we use an approximation argument to generalize Corollary 3.7 to Theorem 3.1.

Proof of Theorem 3.1. Fix $1 \leq k \leq n$. Under our assumptions on $\mathcal{S}$, Lipschitz mappings form a dense subset of $N_{\text {loc }}^{1, n}\left(\mathcal{S}, \mathbf{R}^{n}\right)$ and thus by Corollary 3.7 is suffices to show that if $\left\{f^{j}\right\}_{j=1}^{\infty}$ is a sequence of Lipschitz mappings converging to $f \in N_{\mathrm{loc}}^{1, n}(\Omega)$, then

$$
\int_{\Omega} \eta J_{f^{j}} d \mathcal{H}^{n} \rightarrow \int_{\Omega} \eta J_{f} d \mathcal{H}^{n}
$$

and

$$
\int_{\Omega} f_{k}^{j} J\left(f_{1}^{j}, \ldots, f_{k-1}^{j}, \eta, f_{k+1}^{j}, \ldots, f_{n}^{j}\right) d \mathcal{H}^{n} \rightarrow \int_{\Omega} f_{k} J\left(f_{1}, \ldots, f_{k-1}, \eta, f_{k+1}, \ldots, f_{n}\right) d \mathcal{H}^{n}
$$


as $j \rightarrow \infty$. The first convergence follows directly from [10, Corollary 2.31]. For the second, we need to show that

$$
\int_{\Omega}\left(f_{k}^{j}-f_{k}\right) J\left(f_{1}^{j}, \ldots, f_{k-1}^{j}, \eta, f_{k+1}^{j}, \ldots, f_{n}^{j}\right) d \mathcal{H}^{n} \rightarrow 0
$$

and

(6) $\int_{\Omega} f_{k}\left(J\left(f_{1}^{j}, \ldots, f_{k-1}^{j}, \eta, f_{k+1}^{j}, \ldots, f_{n}^{j}\right)-J\left(f_{1}, \ldots, f_{k-1}, \eta, f_{k+1}, \ldots, f_{n}\right)\right) d \mathcal{H}^{n} \rightarrow 0$.

Here,

$$
\begin{aligned}
& \left|\int_{\Omega}\left(f_{k}^{j}-f_{k}\right) J\left(f_{1}^{j}, \ldots, f_{k-1}^{j}, \eta, f_{k+1}^{j}, \ldots, f_{n}^{j}\right) d \mathcal{H}^{n}\right| \\
& \leq\left(\int_{\Omega}\left|f_{k}^{j}-f_{k}\right|^{n} d \mathcal{H}^{n}\right)^{\frac{1}{n}} \operatorname{Lip} \eta\left(\int_{\Omega}\left\|D f^{j}\right\|^{n} d \mathcal{H}^{n}\right)^{\frac{n-1}{n}} \rightarrow 0,
\end{aligned}
$$

as $f^{j} \rightarrow f$ in $N_{\text {loc }}^{1, n}\left(\Omega, \mathbf{R}^{n}\right)$. On the other hand,

$$
\begin{aligned}
& \left|\int_{\Omega} f_{k}\left(J\left(f_{1}^{j}, \ldots, f_{k-1}^{j}, \eta, f_{k+1}^{j}, \ldots, f_{n}^{j}\right)-J\left(f_{1}, \ldots, f_{k-1}, \eta, f_{k+1}, \ldots, f_{n}\right)\right) d \mathcal{H}^{n}\right| \\
& =\left|\int_{\Omega} f_{k} \sum_{i \neq k} J\left(f_{1}^{j}, f_{2}^{j}, \ldots, f_{i-1}^{j}, f_{i}^{j}-f_{i}, f_{i+1}, \ldots, f_{k-1}, \eta, f_{k+1}, \ldots, f_{n}\right) d \mathcal{H}^{n}\right| \\
& \leq \operatorname{Lip} \eta \int_{\Omega}\left|f_{k}\right|\left(\left\|D f^{j}\right\|+\|\operatorname{ap} D f\|\right)^{n-2}\left\|\operatorname{ap} D\left(f^{j}-f\right)\right\| d \mathcal{H}^{n} \\
& \leq \operatorname{Lip} \eta\left(\int_{\Omega}\left|f_{k}\right|^{n} d \mathcal{H}^{n}\right)^{\frac{1}{n}}\left(\int_{\Omega}\left(\left\|D f^{j}\right\|+\|\operatorname{ap} D f\|\right)^{n} d \mathcal{H}^{n}\right)^{\frac{n-2}{n}} \\
& \quad \cdot\left(\int_{\Omega}\left\|\operatorname{ap} D\left(f^{j}-f\right)\right\|^{n} d \mathcal{H}^{n}\right)^{\frac{1}{n}} \rightarrow 0,
\end{aligned}
$$

by (4) as $j \rightarrow \infty$. Thus the claim follows.

\section{Higher integrability, continuity and compactness}

In this section we prove higher integrability for the Jacobian of quasiregular mappings. The integration by parts formula of Theorem 3.1 is a crucial tool here. We start with the definition of quasiregular map.

Definition 4.1. We say that $f \in N_{\text {loc }}^{1, n}\left(\mathcal{S}, \mathbf{R}^{n}\right)$ is quasiregular if there exists a constant $K \geq 1$ such that

$$
\|\operatorname{ap} D f(x)\|^{n} \leq K J_{f}(x)
$$

for $\mathcal{H}^{n}$-almost every $x \in \mathcal{S}$.

Remark 4.2. The smallest constant $K \geq 1$ satisfying (7) is called outer distortion and denoted by $K_{O}$. It is a linear algebraic fact that

$$
\frac{\left\|\operatorname{ap} D^{\sharp} f(x)\right\|^{n}}{J_{f}(x)^{n-1}} \leq K_{O}^{n-1},
$$

whenever $J_{f}(x)>0$. Here $\operatorname{ap} D^{\sharp} f(x)$ is the adjoint matrix of ap $D f(x)$. The smallest constant dominating the ratio above is called inner distortion and denoted by $K_{I}$. Thus $K_{I} \leq K_{O}^{n-1}$. Conversely, we have $K_{O} \leq K_{I}^{n-1}$. It follows from Hadamard's 
inequality $J_{f}(x) \leq\|D f(x)\|^{n}$ that the Jacobian of a quasiregular map is locally integrable. The higher integrability Theorem 4.4 gives an improvement for this. If we wish to emphasise that a quasiregular map $f$ satisfies $(7)$ with some specific $K$ we say that $f$ is $K$-quasiregular.

The following Caccioppoli estimate turns out to be useful in proofs of the higher integrability Theorem 4.4 and the compactness Theorem 4.6.

Lemma 4.3. Assume that $f: \mathcal{S} \rightarrow \mathbf{R}^{n}$ is a quasiregular map. Let $B=B(z, r)$ and let $\eta \in \operatorname{Lip}_{0}(2 B)$ be a non-negative $\frac{1}{r}$-Lipschitz function. Then

$$
\left(\int_{2 B} \eta^{n} J_{f} d \mathcal{H}^{n}\right)^{\frac{1}{n}} \leq C K^{\frac{n-1}{n}}\left(f_{2 B}\left|f_{k}-\left(f_{k}\right)_{2 B}\right|^{n} d \mathcal{H}^{n}\right)^{\frac{1}{n}}
$$

for each component function $f_{k}$ of $f$. Here $\left(f_{k}\right)_{2 B}=f_{2 B} f_{k} d \mathcal{H}^{n}$.

Proof. We may assume that $k=1$. Let us write $g=\left(f_{1}-\left(f_{1}\right)_{2 B}, f_{2}, \ldots, f_{n}\right)$. By Theorem 3.1 we have

$$
\begin{aligned}
& \left|\int_{2 B} \eta^{n} J_{f} d \mathcal{H}^{n}\right|=\left|\int_{2 B} \eta^{n} J_{g} d \mathcal{H}^{n}\right|=\left|\int_{2 B} g_{1} J\left(\eta^{n}, g_{2}, \ldots, g_{n}\right) d \mathcal{H}^{n}\right| \\
& =\left|\int_{2 B} n g_{1} \eta^{n-1} J\left(\eta, f_{2}, \ldots, f_{n}\right) d \mathcal{H}^{n}\right| \leq C \int_{2 B}\left|g_{1}\right| n \eta^{n-1} \frac{1}{r}\|\operatorname{ap} D f\|^{n-1} d \mathcal{H}^{n} .
\end{aligned}
$$

By the distortion inequality (7) and Hölder's inequality, we get

$$
\int_{2 B}\left|g_{1}\right| n \eta^{n-1} \frac{1}{r}\|\operatorname{ap} D f\|^{n-1} d \mathcal{H}^{n} \leq C K^{\frac{n-1}{n}}\left(\int_{2 B} \eta^{n} J_{f} d \mathcal{H}^{n}\right)^{\frac{n-1}{n}}\left(f_{2 B}\left|g_{1}\right|^{n} d \mathcal{H}^{n}\right)^{\frac{1}{n}} \text {. }
$$

This together with (8) gives the claim.

Theorem 4.4. For every quasiregular map $f: \mathcal{S} \rightarrow \mathbf{R}^{n}$ there exists $\epsilon>0$ depending on $n, K$ and $\mathcal{S}$ such that $J_{f} \in L_{\text {loc }}^{1+\epsilon}(\mathcal{S})$. Furthermore there is a constant $\sigma \geq 1$ such that

$$
\left(f_{B} J_{f}(x)^{1+\epsilon} d \mathcal{H}^{n}(x)\right)^{\frac{1}{1+\epsilon}} \leq C(\mathcal{S}, K) f_{\sigma B} J_{f}(x) d \mathcal{H}^{n}(x)
$$

for every ball $B \subset \sigma B \subset \mathcal{S}$.

Proof. For a Lipschitz map $f,\|D f(\cdot)\|$ is $n$-weak upper gradient and thus $f_{1}$ and $\|D f(\cdot)\|$ satisfy the Sobolev-Poincaré inequality (2). Therefore it follows from inequality (4) and density of Lipschitz mappings that for $f \in N_{\text {loc }}^{1, n}\left(\mathcal{S}, \mathbf{R}^{n}\right), f_{1}$ and $\|\operatorname{ap} D f(\cdot)\|$ satisfy the Sobolev-Poincaré inequality (2). This together with the distortion inequality $(7)$ gives

$$
\begin{aligned}
\left(f_{2 B}\left|f_{1}-\left(f_{1}\right)_{2 B}\right|^{n} d \mathcal{H}^{n}\right)^{\frac{1}{n}} & \leq C r\left(f_{2 \tau B}\|\operatorname{ap} D f\|^{\frac{n}{2}} d \mathcal{H}^{n}\right)^{\frac{2}{n}} \\
& \leq C r K^{\frac{1}{n}}\left(f_{2 \tau B} J_{f}^{\frac{1}{2}} d \mathcal{H}^{n}\right)^{\frac{2}{n}} .
\end{aligned}
$$

Now one can choose $\eta \in \operatorname{Lip}_{0}(2 B)$ in Lemma 4.3 such that $\eta_{\mid B}=1$ to obtain the following reverse Hölder's inequality

$$
\left(f_{B} J_{f}(x) d \mathcal{H}^{n}(x)\right)^{\frac{1}{n}} \leq C K\left(f_{2 \tau B} J_{f}(x)^{\frac{1}{2}} d \mathcal{H}^{n}(x)\right)^{\frac{2}{n}} .
$$


Thus the claim follows directly from a version of Gehring Lemma given in [20, Theorem 3.3].

As a corollary of Theorem 4.4 we get the following.

Corollary 4.5. Let $f: \mathcal{S} \rightarrow \mathbf{R}^{n}$ be quasiregular. Then $f \in N_{\text {loc }}^{1, p}\left(\mathcal{S}, \mathbf{R}^{n}\right)$ for some $p>n$. In particular $f$ is locally Hölder continuous and

$$
|f(x)-f(y)| \leq C r^{\frac{n}{p}}|x-y|^{1-\frac{n}{p}}\left(f_{5 \sigma B(z, r)}\|D f(x)\|^{p} d \mathcal{H}^{n}(x)\right)^{\frac{1}{p}},
$$

for every $x, y \in B(z, r)$. Here $C$ and $\sigma$ depend only on the space $\mathcal{S}$ and on $p$.

Proof. It follows from Theorem 4.4 that if $f \in N_{\text {loc }}^{1, n}\left(\mathcal{S}, \mathbf{R}^{n}\right)$ satisfies the distortion inequality (7) then $\|D f\| \in L_{\text {loc }}^{1, p}(\mathcal{S})$ for some $p>n$. Thus by [3, Theorem 5.1] $f$ is locally Hölder continuous and the given modulus of continuity holds.

Next we turn to the following compactness theorem for quasiregular mappings.

Theorem 4.6. Let $\Omega \subset \mathcal{S}$ be a domain. If a sequence $\left\{f^{i}\right\}_{i=1}^{\infty}$ of $K$-quasiregular mappings, $f^{i}: \Omega \rightarrow \mathbf{R}^{n}$ converges locally uniformly, then also the limiting map is $K$-quasiregular.

In the Euclidean case a similar convergence result is due Reshetnyak, see [14]. A simplified version of the proof is given by Lindqvist in [11], see also [16, VI 8].

Lemma 4.7. Let $\Omega \subset \mathcal{S}$ be a domain and let $\left\{f^{i}\right\}_{i=1}^{\infty}$ be a sequence of $K$ quasiregular mappings, $f^{i}: \Omega \rightarrow \mathbf{R}^{n}$ that converges locally uniformly to a limiting map $f$. Then $\left\{f^{i}\right\}_{i=1}^{\infty}$ has a subsequence still denoted by $\left\{f^{i}\right\}_{i=1}^{\infty}$ such that $\left\{\left\|D f^{i}\right\|\right\}_{i=1}^{\infty}$ converges weakly in $L^{p}(\Omega)$ to function $g$ that is an $n$-weak upper gradient of $f$. In particular $f \in N^{1, n}\left(\Omega, \mathbf{R}^{n}\right)$.

Proof. We employ Lemma 4.3 again as in the proof of Theorem 4.4 together with the distortion inequality to obtain

$$
\int_{B}\left\|D f^{i}(x)\right\|^{n} d \mathcal{H}^{n}(x) \leq C(K)\left(f_{2 B}\left|f_{1}^{i}(x)-\left(f_{1}^{i}\right)_{2 B}\right|^{n} d \mathcal{H}^{n}(x)\right)^{\frac{1}{n}} .
$$

Therefore it follows from uniform convergence that for any compact set $\Omega^{\prime} \subset \Omega$ there exists a constant $C\left(\Omega^{\prime}, K\right)$ such that

$$
\int_{\Omega^{\prime}}\left\|D f^{i}(x)\right\|^{n} d \mathcal{H}^{n}(x) \leq C\left(\Omega^{\prime}, K\right)
$$

for every $i$. We conclude that for any compact $\Omega^{\prime} \subset \Omega$ the sequence $\left\{\left\|D f^{i}\right\|\right\}_{i=1}^{\infty}$ is bounded in $L^{n}\left(\Omega^{\prime}\right)$ and therefore it has a subsequence converging weakly to a function $g$. By [18, Lemma 4.11] $g$ is an $n$-weak upper gradient of the uniform limit $f=\lim _{i} f^{i}$. Therefore it follows that $f \in N_{\text {loc }}^{1, n}\left(\Omega, \mathbf{R}^{n}\right)$.

Due to the integration by parts formula given in Theorem 3.1, Lemma 4.8 can be proved as in the Euclidean case up to some obvious modifications, see [11, Lemma 4.2] or [16, VI. Lemma 8.8].

Lemma 4.8. Let $\Omega \subset \mathcal{S}$ be a relatively compact open set and let $\left\{f_{i}\right\}_{i=1}^{\infty}$ be a sequence of mappings in $N^{1, n}\left(\Omega, \mathbf{R}^{n}\right)$ converging uniformly to a map $f \in N^{1, n}\left(\Omega, \mathbf{R}^{n}\right)$. Let $g \in N^{1, n}\left(\Omega, \mathbf{R}^{n}\right)$ and $\eta \in \operatorname{Lip}_{0}(\Omega)$. Then for any $1 \leq p \leq n$ we have

$$
\int_{\Omega} \eta^{p} J\left(f_{1}^{i}, f_{2}^{i}, \ldots, f_{p}^{i}, g_{p+1}, \ldots, g_{n}\right) d \mathcal{H}^{n} \rightarrow \int_{\Omega} \eta^{p} J\left(f_{1}, f_{2}, \ldots, f_{p}, g_{p+1}, \ldots, g_{n}\right) d \mathcal{H}^{n}
$$


when $i \rightarrow \infty$.

Now we are ready to prove Theorem 4.6, assuming Proposition 4.9 below.

Proof of Theorem 4.6. By Lemma 4.7 we are left to prove the distortion inequality (7) for the limit mapping $f$. Let $g$ be as in Lemma 4.7. Fix $\epsilon>0$ and $x \in \Omega$ such that $x$ is a Lebesgue point of both $J_{f}$ and $g^{n}$. For any $B(x, r+\epsilon) \subset \Omega$, and for $\eta \in \operatorname{Lip}_{0}(B(x, r+\epsilon))$ satisfying $0 \leq \eta \leq 1$ and $\eta_{\mid B(x, r)}=1$, we apply weak semicontinuity of the $L^{n}$-norm, distortion inequality (7), and Lemma 4.8 to find

$$
\begin{aligned}
& \int_{B(x, r)} g(z)^{n} d \mathcal{H}^{n} \leq \liminf _{i \rightarrow 0} \int_{B(x, r)}\left\|\operatorname{ap} D f_{i}(z)\right\|^{n} d \mathcal{H}^{n} \\
& \leq \liminf _{i \rightarrow 0} \int_{B(x, r+\epsilon)}\left\|\operatorname{ap} D f_{i}(z)\right\|^{n} \eta(z)^{n} d \mathcal{H}^{n} \leq K \liminf _{i \rightarrow 0} \int_{B(x, r+\epsilon)} J_{f_{i}}(z) \eta(z)^{n} d \mathcal{H}^{n} \\
& =K \int_{B(x, r+\epsilon)} J_{f}(z) \eta(z)^{n} d \mathcal{H}^{n} \leq K \int_{B(x, r+\epsilon)} J_{f}(z) d \mathcal{H}^{n} .
\end{aligned}
$$

Letting $\epsilon \rightarrow 0$ and applying the Lebesgue differentiation theorem we obtain

$$
g(x)^{n} \leq K J_{f}(x)
$$

Now since $\|\operatorname{ap} D f(\cdot)\|$ is a minimal upper garadient of $f$ in the sense of inequality (4) we have

$$
\|\operatorname{ap} D f(x)\|^{n} \leq C(\mathcal{S}) K J_{f}(x),
$$

for $\mathcal{H}^{n}$-almost every $x \in \Omega$. Therefore $f$ is $C(\mathcal{S}) K$-quasiregular. In order to find that $f$ is actually $K$-quasiregular we apply [10, Theorem 6.3] to see that $f$ is differentiable the $\mathcal{H}^{n}$-almost everywhere. Then Proposition 4.9 together with (10) gives the desired distortion inequality and thus $f$ is $K$-quasiregular.

Proposition 4.9. Assume that $f: \mathcal{S} \rightarrow \mathbf{R}^{n}$ is differentiable $\mathcal{H}^{n}$-almost everywhere. Let $p \geq 1$ and let $g$ be an integrable $p$-weak upper gradient of $f$. Then $\|D f(x)\| \leq g(x)$, for $\mathcal{H}^{n}$-almost every $x \in \mathcal{S}$.

In order to prove Proposition 4.9 we need some preparations.

Lemma 4.10. Let $x_{0} \in \mathcal{S}$ and let $Q \subset T_{x_{0}} \mathcal{S}$ be a $(n-1)$-dimensional cube with side length 1. Let $B^{+}$and $B^{-}$be two faces of $Q$ parallel to each other, and let $K \subset \mathcal{S}$ be a compact set. Then

(1) For $\mathcal{H}^{1}$-almost every $a>0$ the following holds. For $\mathcal{H}^{n-1}$-almost every $y \in$ $a B^{+}$the multiplicity card $\pi_{x_{0}}^{-1}(y) \cap K$ is finite.

(2) For every $a>0$ the following holds. For each $s \in a B^{+}$let $J_{s}$ be the line segment perpendicular to $B^{+}$, starting at $s \in a B^{+}$and ending at $a B^{-}$. Then for $\mathcal{H}^{n-1}$-almost every $s \in a B^{+}$we have $\mathcal{H}^{1}\left(\pi_{x_{0}}^{-1}\left(J_{s}\right) \cap K\right)<\infty$.

Recall that $\pi_{x_{0}}$ is the orthogonal projection from $\mathcal{S}$ to the approximate tangent plane $T_{x_{0}} \mathcal{S}$.

Proof. To prove the first claim, assume to the contrary that the $\mathcal{H}^{1}$-measure of the set

$$
P=\left\{a>0: \exists C_{a} \subset a B^{+}, \mathcal{H}^{n-1}\left(C_{a}\right)>0 \text { and } \operatorname{card} \pi_{x_{0}}^{-1}(y) \cap K=\infty \forall y \in C_{a}\right\}
$$

is positive. Then

$$
\int_{P} \int_{C_{a}} \operatorname{card} \pi_{x_{0}}^{-1}(y) \cap K d \mathcal{H}^{n-1}(y) d \mathcal{H}^{1}(a)=\infty .
$$


On the other hand, since $\pi_{x_{0}}$ is 1-Lipschitz, it follows from the coarea formula of Theorem 3.3 that

$$
\begin{aligned}
\infty & >\int_{K}\left|J_{\pi_{x_{0}}}\right| d \mathcal{H}^{n} \geq \int_{\cup_{a \in P} C_{a}} \operatorname{card} \pi_{x_{0}}^{-1}(y) \cap K d \mathcal{H}^{n}(y) \\
& =\int_{P} \int_{C_{a}} \operatorname{card} \pi_{x_{0}}^{-1}(y) \cap K d \mathcal{H}^{n-1}(y) d \mathcal{H}^{1}(a) .
\end{aligned}
$$

This is a contradiction and thus the first part of the claim holds. To prove the second claim fix $a>0$ and let pr: $T_{x_{0}} \mathcal{S} \rightarrow a B^{+}$be the orthogonal projection that maps $J(s)$ to $s$. Then $\operatorname{pr} \circ \pi$ is Lipschitz and again by the coarea formula

$$
\begin{aligned}
\int_{a B^{+}} \mathcal{H}^{1}\left(\pi_{x_{0}}^{-1}\left(J_{s}\right) \cap K\right) d \mathcal{H}^{n-1}(s) & =\int_{a B^{+}} \int_{\left(\operatorname{pro} \pi_{x_{0}}\right)^{-1}(s) \cap K} 1 d \mathcal{H}^{1} d \mathcal{H}^{n-1}(s) \\
& \leq \int_{K}\left|J_{\operatorname{pr} \circ \pi_{x_{0}}}\right| d \mathcal{H}^{n}<\infty
\end{aligned}
$$

This proves the lemma.

Lemma 4.11. Let $x_{0} \in \mathcal{S}$ such that $T_{x_{0}} \mathcal{S}$ exists and

$$
\lim _{r \rightarrow 0} \frac{\mathcal{H}^{n}\left(B\left(x_{0}, r\right) \cap \mathcal{S}\right)}{\alpha_{n} r^{n}}=1 .
$$

Let $Q_{r} \subset T_{x_{0}} \mathcal{S}$ be an $n$-dimensional cube with side length $r>0$ centered at $x_{0}$. Then

$$
\lim _{r \rightarrow 0} \frac{\mathcal{H}^{n}\left(\pi_{x_{0}}^{-1}\left(Q_{r}\right)\right)}{\mathcal{H}^{n}\left(Q_{r}\right)}=1
$$

Proof. Let us first prove (11) in the case that $Q_{r}$ is replaced by $B\left(x_{0}, r\right) \cap T_{x_{0}} \mathcal{S}$. We may assume that $x_{0}=0$. For $z \in \mathcal{S}$ we write

$$
\left(\pi_{0} z\right)^{\perp}=z-\pi_{0} z \in \mathbf{R}^{m} .
$$

It follows from [10, Corollary 2.13] that for the function $\epsilon:(0, \infty) \rightarrow(0, \infty)$,

$$
\epsilon(r)=\sup _{|z| \leq r} \frac{\left|\left(\pi_{0} z\right)^{\perp}\right|}{\left|\pi_{0} z\right|},
$$

$\lim _{r \rightarrow 0} \epsilon(r)=0$. Now, by triangle inequality, $\frac{|z|}{\left|\pi_{0} z\right|} \leq 1+\epsilon(|z|)$ and therefore $(1-$ $\epsilon(|z|))|z| \leq\left|\pi_{0} z\right|$ whenever $x \in \mathcal{S}$. Fix $r>0$ so small that $|z| \leq 2\left|\pi_{0} z\right|$ for every $z \in B(0, r) \cap \mathcal{S}$ and let $y \in B(0, r) \cap T_{0} \mathcal{S}$. Now if $\pi_{0} z=y$ we have

$$
(1-\epsilon(r))|z| \leq(1-\epsilon(|z|))|z| \leq\left|\pi_{0} z\right|=|y| \leq r .
$$

Therefore $|z| \leq \frac{r}{1-\epsilon(r)}$. That is $\pi_{0}^{-1}(B(0, r)) \subset B\left(0, \frac{r}{1-\epsilon(r)}\right)$. Since $\pi_{0}$ is 1-Lipschitz, we have

$$
\begin{aligned}
1 & \leq \frac{\mathcal{H}^{n}\left(\pi_{0}^{-1}(B(0, r))\right)}{\mathcal{H}^{n}\left(B(0, r) \cap T_{0} \mathcal{S}\right)} \leq \frac{\mathcal{H}^{n}\left(B\left(0, \frac{r}{1-\epsilon(r)}\right) \cap \mathcal{S}\right)}{\mathcal{H}^{n}\left(B(0, r) \cap T_{0} \mathcal{S}\right)} \\
& \leq \frac{\alpha_{n} r^{n}}{\mathcal{H}^{n}\left(B(0, r) \cap T_{0} \mathcal{S}\right)} \frac{\left(\frac{r}{1-\epsilon(r)}\right)^{n}}{r^{n}} \frac{\mathcal{H}^{n}\left(B\left(0, \frac{r}{1-\epsilon(r)}\right) \cap \mathcal{S}\right)}{\alpha_{n}\left(\frac{r}{1-\epsilon(r)}\right)^{n}} .
\end{aligned}
$$

Because $x_{0}=0$ was assumed to be a point of density one this gives (11) for $B\left(x_{0}, r\right) \cap$ $T_{x_{0}} \mathcal{S}$. To prove (11) for $Q_{r}$, note first that $B\left(x_{0}, r\right) \cap T_{x_{0}} \mathcal{S} \subset Q_{r} \subset B\left(x_{0}, \sqrt{n} r\right) \cap T_{x_{0}} \mathcal{S}$. 
Again since $\pi_{x_{0}}$ is 1-Lipschitz, $1 \leq \frac{\mathcal{H}^{n}\left(\pi_{x_{0}}^{-1}\left(Q_{r}\right)\right)}{\mathcal{H}^{n}\left(Q_{r}\right)}$. On the other hand, if there is $\delta>0$ and arbitrary small radii $r$ such that

$$
\mathcal{H}^{n}\left(\pi_{x_{0}}^{-1}\left(Q_{r}\right)\right) \geq(1+\delta) \mathcal{H}^{n}\left(Q_{r}\right)
$$

we get

$$
\begin{aligned}
\mathcal{H}^{n}\left(\pi_{x_{0}}^{-1}\left(B\left(x_{0}, \sqrt{n} r\right)\right)\right) & =\mathcal{H}^{n}\left(\pi_{x_{0}}^{-1}\left(B\left(x_{0}, \sqrt{n} r\right) \backslash Q_{r}\right)\right)+\mathcal{H}^{n}\left(\pi_{x_{0}}^{-1}\left(Q_{r}\right)\right) \\
& \geq \mathcal{H}^{n}\left(B\left(x_{0}, \sqrt{n} r\right) \cap T_{x_{0}} \mathcal{S} \backslash Q_{r}\right)+(1+\delta) \mathcal{H}^{n}\left(Q_{r}\right) \\
& \geq \mathcal{H}^{n}\left(B\left(x_{0}, \sqrt{n} r\right) \cap T_{x_{0}} \mathcal{S}\right)+\delta \mathcal{H}^{n}\left(B\left(x_{0}, r\right) \cap T_{x_{0}} \mathcal{S}\right) .
\end{aligned}
$$

That is

$$
\frac{\mathcal{H}^{n}\left(\pi_{0}^{-1}\left(B\left(x_{0}, \sqrt{n} r\right)\right)\right)}{\mathcal{H}^{n}\left(B\left(x_{0}, \sqrt{n} r\right) \cap T_{x_{0}} \mathcal{S}\right)} \geq 1+\delta \frac{\mathcal{H}^{n}\left(B\left(x_{0}, r\right) \cap T_{x_{0}} \mathcal{S}\right)}{\mathcal{H}^{n}\left(B\left(x_{0}, \sqrt{n} r\right) \cap T_{x_{0}} \mathcal{S}\right)}
$$

However, since (11) holds for $B\left(x_{0}, r\right) \cap T_{x_{0}} \mathcal{S}$ this is impossible for sufficiently small radii. Therefore $\delta>0$ as above does not exist and the lemma is proven.

Lemma 4.12. Assume that $x_{0} \in \mathcal{S}$ such that $T_{x_{0}} \mathcal{S}$ exists and let $U$ be a sufficiently small neighborhood of $x_{0}$. Assume that $J$ is a path in $T_{x_{0}}$ such that its trace $|J|$ is a line segment $[a, b]$ in the $x_{0}$-component of $\pi_{x_{0}}(\partial U)^{c}$. In addition assume that card $\pi_{x_{0}}^{-1}(a) \cap \bar{U}$ and $\mathcal{H}^{1}\left(\pi_{x_{0}}^{-1}(|J|) \cap \bar{U}\right)$ are finite. Then there exists a rectifiable injective path $\gamma:[0,1] \rightarrow U$ such that $\left(\pi_{x_{0}} \circ \gamma\right)(0)=a,\left(\pi_{x_{0}} \circ \gamma\right)(1)=b$ and $\left|\pi_{x_{0}} \circ \gamma\right|=|J|$.

Proof. Since $a$ is in the $x_{0}$-component of $\pi(\partial U)^{c}$ and $U$ is a small neighborhood of $x_{0}$ it follows from the metric orientation and from basic properties of topological degree, see Lemma 2.7, that $1=\mu\left(x_{0}, \pi_{x_{0}}, U\right)=\mu\left(a, \pi_{x_{0}}, U\right)$. Let $\left\{C_{1}, C_{2}, \ldots, C_{p}\right\}$ be the collection of the components of $\pi_{x_{0}}^{-1}(|J|) \cap U$ that includes a point from $\pi_{x_{0}}^{-1}(a)$. We find disjoint domains $U_{1}, U_{2}, \ldots, U_{p} \subset U$ each including one of the components $C_{i}$. By Lemma 2.7 such domains satisfy $1=\mu\left(a, \pi_{x_{0}}, U\right)=\sum_{i=1}^{p} \mu\left(a, \pi_{x_{0}}, U_{i}\right)$, and therefore for some $i=i_{0}$ we have $\mu\left(a, \pi_{x_{0}}, U_{i_{0}}\right) \geq 1$. In particular, the domains $U_{i}$ can be chosen to belong to an arbitrary small neighborhoods of the corresponding components $C_{i}$.

If $\pi_{x_{0}}^{-1}(|J|) \cap \partial U_{i_{0}}=\emptyset$, write $V=U_{i_{0}}$. If $\pi_{x_{0}}^{-1}(|J|) \cap \partial U_{i_{0}} \neq \emptyset$ and $\pi_{x_{0}}^{-1}(|J|) \cap U_{i_{0}}=$ $C_{i_{0}}$, we find another domain $W$ satisfying $C_{i_{0}} \subset W \subset U_{i_{0}}$ and $\pi_{x_{0}}^{-1}(|J|) \cap \partial W=\emptyset$. In this case write $V=W$.

Finally, in the case that $\pi_{x_{0}}^{-1}(|J|) \cap \partial U_{i_{0}} \neq \emptyset$ and $\left(\pi_{x_{0}}^{-1}(|J|) \cap U_{i_{0}}\right) \backslash C_{i_{0}} \neq \emptyset$, the set $\pi_{x_{0}}^{-1}(|J|) \cap U_{i_{0}}$ is disconnected in $U_{i_{0}}$ and therefore we find open disjoint sets $A_{1}, A_{2} \subset$ $U_{i_{0}}$ satisfying $\pi_{x_{0}}^{-1}(|J|) \cap U_{i_{0}} \subset A_{1} \cup A_{2}$ and $C_{i_{0}} \subset A_{1}$. Now, $\pi_{x_{0}}^{-1}(|J|) \cap \partial A_{1}=\emptyset$ and we choose $V$ to be the component of $A_{1}$ that includes $C_{i_{0}}$.

Thus, in all the cases, we have found domain $V \subset U$ such that $C_{i_{0}} \subset V$ and $\pi_{x_{0}}^{-1}(|J|) \cap \partial V=\emptyset$. Furthermore, because $\pi_{x_{0}}^{-1}(a) \cap U_{i_{0}} \subset C_{i_{0}} \subset V \subset U_{i_{0}}$ we have $\mu\left(a, \pi_{x_{0}}, V\right)=\mu\left(a, \pi_{x_{0}}, U_{i_{0}}\right) \geq 1$ and thus because $|J|$ is in the $a$-component of $\pi(\partial V)^{c}$ we have $\mu\left(p, \pi_{x_{0}}, V\right)=\mu\left(a, \pi_{x_{0}}, V\right) \geq 1$ for every $p \in|J|$. Therefore $|J| \subset \pi_{x_{0}}(V)$. Furthermore, since the sets $U_{i}$ can be chosen to be arbitrarily small neighborhoods of the sets $C_{i}$ and because $\pi_{x_{0}}$ is continuous we conclude that $|J|=\pi_{x_{0}} C_{i_{0}}$. Since $C_{i_{0}}$ is compact, connected and satisfies $\mathcal{H}^{1}\left(C_{i_{0}}\right)<\infty$, it follows from [17, Proposition 15.1] that for each pair or points in $C_{i_{0}}$ there is a injective rectifiable path connecting them. In particular, if we choose one point from $\pi_{x_{0}}^{-1}(a) \cap C_{i_{0}}$ and another from $\pi_{x_{0}}^{-1}(b) \cap C_{i_{0}}$, we find the desired path.

Now we are ready to prove Proposition 4.9. 
Proof of Proposition 4.9. By [10, Definition 2.26], we may consider only those $x \in$ $\mathcal{S}$ satisfying the following. Let $x_{0}$ be a point of differentiability such that $\left\|D f\left(x_{0}\right)\right\|=$ $\left|D f(0) y_{0}\right|$, for some $y_{0} \in T_{x_{0}} \mathcal{S}$ with $\left|y_{0}\right|=1$. Furthermore, we may assume that $x_{0}$ is a Lebesgue point of $g$ and $\lim _{r \rightarrow 0} \frac{\mathcal{H}^{n}\left(B\left(x_{0}, r\right)\right)}{\alpha_{n} r^{n}}=1$. In addition, by translating we may assume that $x_{0}$ is the origin of the ambient space $\mathbf{R}^{m}$ and $f(0)=0$. Let $U$ be a small neighborhood of 0 as in the proof of Lemma 4.12. Let $a>0$. Let $A^{\prime}=\left\{x \in T_{x_{0}} \mathcal{S}: \quad\left\langle x, y_{0}\right\rangle=0\right\}$ and let $A$ be a $(n-1)$-dimensional cube in $A^{\prime}$ centered at origin and with side length $2 a$. Let $B^{+}=A+a y_{0}$ and $B^{-}=A-a y_{0}$. For each $s \in A$ let $J_{s}$ be the line segment connecting $s-a y_{0}$ to $s+a y_{0}$ and let $Q_{a}$ be the $n$-dimensional cube $\bigcup_{s \in A} J_{s}$. We assume that $a$ is chosen such that $Q_{a}$ is in the 0-component of $\pi_{0}(\partial U)^{c}$ and that for $\mathcal{H}^{n-1}$-almost every $s \in \mathcal{S}$ the line $J_{s}$ satisfies card $\pi_{0}^{-1}\left(s-a y_{0}\right) \cap U<\infty$ and $\mathcal{H}^{1}\left(\pi_{0}^{-1}(|J|) \cap U\right)<\infty$. Note that by Lemma 4.10 $\mathcal{H}^{1}$-almost every small $a>0$ satisfies these conditions. According to Lemma 4.12 for $\mathcal{H}^{n-1}$-almost every line $J_{s}$ there is a injective rectifiable path $\gamma_{s}:[0,1] \rightarrow U$ such that $\left(\pi_{0} \circ \gamma_{s}\right)(0)=s-a y_{0},\left(\pi_{0} \circ \gamma_{s}\right)(1)=s+a y_{0}$, and $\left|\left(\pi_{0} \circ \gamma_{s}\right)\right|=J_{s}$. Denote $\gamma_{s}(0)=\left(s-a y_{0}\right)^{\prime}$ and $\gamma_{s}(1)=\left(s+a y_{0}\right)^{\prime}$. Then $\pi_{0}\left(\left(s-a y_{0}\right)^{\prime}\right)=s-a y_{0}$ and $\pi_{0}\left(\left(s+a y_{0}\right)^{\prime}\right)=s+a y_{0}$.

Because $f$ is differentiable at $0 \in \mathcal{S}$ and $f(0)=0$ we have

$$
f(x)-D f(0) \pi_{0} x=\epsilon(|x|),
$$

where $\frac{\epsilon(|x|)}{|x|} \rightarrow 0$, as $x \rightarrow 0$. Therefore

$$
\begin{aligned}
f\left(\left(s-a y_{0}\right)^{\prime}\right)-f\left(\left(s+a y_{0}\right)^{\prime}\right) & =D f(0)\left(2 a y_{0}\right)+\epsilon\left(\left|s-a y_{0}\right|\right)-\epsilon\left(\left|s+a y_{0}\right|\right) \\
& =2 a\|D f(0)\|+\epsilon\left(\left|s-a y_{0}\right|\right)-\epsilon\left(\left|s+a y_{0}\right|\right) .
\end{aligned}
$$

That is,

$$
2 a\|D f(0)\|+\epsilon\left(\left|s-a y_{0}\right|\right)-\epsilon\left(\left|s+a y_{0}\right|\right)=f\left(\left(s-a y_{0}\right)^{\prime}\right)-f\left(\left(s+a y_{0}\right)^{\prime}\right) .
$$

Next we show that $f, g$ and $\gamma_{s}$ satisfy the upper gradient inequality (1) for $\mathcal{H}^{n-1}$ almost every $s \in A$. Assume to the contrary that there is set $K \subset A$ with positive $\mathcal{H}^{n-1}$-measure such that the $p$-modulus of the family $\Gamma=\left\{\gamma_{s}: s \in K\right\}$ is zero. Let pr: $Q_{a} \rightarrow A$ be the projection satisfying $\operatorname{pr}\left(s+p y_{0}\right)=s+a y_{0}$. Then pr is 1-Lipschitz and $\left|\gamma_{s}\right| \subset\left(\operatorname{pr} \circ \pi_{0}\right)^{-1}\left(\left(s+a y_{0}\right)^{\prime}\right)$ for $\mathcal{H}^{n-1}$-almost every $s \in K$. Let $\rho \in \operatorname{Adm} \Gamma$. Then $1 \leq \int_{\gamma_{s}} \rho d s \leq \int_{(\operatorname{pro\pi })^{-1}\left(\left(s+a y_{0}\right)^{\prime}\right)} \rho d \mathcal{H}^{1}$ for $\mathcal{H}^{n-1}$-almost every $s \in K$. Therefore by the coarea formula of Theorem 3.3 we have

$$
\begin{aligned}
\mathcal{H}^{n-1}(K) & \leq \int_{K} \int_{(\operatorname{pro\pi })^{-1}\left(\left(s+a y_{0}\right)^{\prime}\right)} \rho d \mathcal{H}^{1} d \mathcal{H}^{n-1}(s) \leq \int_{\bar{U}} \rho\left|J_{\mathrm{pr} \circ \pi_{0}}\right| d \mathcal{H}^{n} \\
& \leq\left(\int_{\bar{U}} \rho^{p} d \mathcal{H}^{n}\right)^{\frac{1}{p}}\left(\int_{\bar{U}}\left|J_{\mathrm{pr} \circ \pi_{0}}\right|^{\frac{p}{p-1}} d \mathcal{H}^{n}\right)^{\frac{p-1}{p}} .
\end{aligned}
$$

Thus

$$
\frac{\left[\mathcal{H}^{n-1}(K)\right]^{p}}{\left(\int_{\bar{U}}\left|J_{\text {pro } \pi_{0}}\right|^{\frac{p}{p-1}} d \mathcal{H}^{n}\right)^{p-1}} \leq \operatorname{Mod}_{p} \Gamma .
$$

Here $\operatorname{pr} \circ \pi_{0}$ is 1-Lipschitz and therefore the denominator is finite and thus we have found a contradiction. Therofore for $\mathcal{H}^{n-1}$-almost every $s \in A f, g$ and $\gamma_{s}$ satisfy the upper gradient inequality. This fact combined with (12) and the coarea formula 
gives

$$
\begin{aligned}
& (2 a)^{n}\|D f(0)\|+\int_{A} \epsilon\left(\left|s-a y_{0}\right|\right)-\epsilon\left(\left|s+a y_{0}\right|\right) d \mathcal{H}^{n-1}(s) \leq \int_{A} \int_{\gamma_{s}} g d \mathcal{H}^{1} d \mathcal{H}^{n-1}(s) \\
& \leq \int_{A} \int_{(\operatorname{pr} \circ \pi)^{-1}\left(\left(s+a y_{0}\right)^{\prime}\right)} g d \mathcal{H}^{1} d \mathcal{H}^{n-1}(s) \leq \int_{\pi_{0}^{-1}\left(Q_{a}\right)} g\left|J_{\operatorname{pr} \circ \pi_{0}}\right| d \mathcal{H}^{n} .
\end{aligned}
$$

Here pr $\circ \pi_{0}$ is 1-Lipschitz and thus $\left|J_{\mathrm{pr} \circ \pi_{0}}\right| \leq 1$. We conclude that

$$
\|D f(0)\| \leq \frac{\mathcal{H}^{n}\left(\pi_{0}^{-1}\left(Q_{a}\right)\right)}{\mathcal{H}^{n}\left(Q_{a}\right)} f_{\pi_{0}^{-1}\left(Q_{a}\right)} g d \mathcal{H}^{n}-f_{A} \frac{\epsilon\left(\left|s-a y_{0}\right|\right)-\epsilon\left(\left|s+a y_{0}\right|\right)}{2 a} d \mathcal{H}^{n-1}(s) .
$$

Since $a>0$ can be arbitrarily small and the last integral converges to 0 , as $a \rightarrow 0$, Lebesgue differentiation theorem together with Lemma 4.11 gives the claim.

\section{Basic properties of branched covers}

A continuous map is called branched cover if it is open and discrete. Recall that a map is open if it maps open sets to open sets, and discrete if the preimage of each point is locally finite. A continuous map $f: \mathcal{S} \rightarrow \mathbf{R}^{n}$ is called sense-preserving if $\mu(y, f, U) \geq 0$ whenever defined, see Section 2. In [10], we proved that a nonconstant quasiregular map form a space satisfying the current axioms to an n-dimensional Euclidean space is a sense-preserving branched cover. In the case $\mathcal{S}=\mathbf{R}^{n}$ this is a celebrated theorem by Reshetnyak. Next we discuss some properties of branched covers.

Definition 5.1. Let $\Omega \subset \mathcal{S}$ be a domain and let $f: \Omega \rightarrow \mathbf{R}^{n}$ be a branched cover. A relatively compact domain $U \subset \Omega$ is called normal domain if $f(\partial U)=$ $\partial f(U)$. Furthermore, a normal domain $U$ is called normal neighborhood of $x \in \mathcal{S}$ if $f^{-1}(f(x)) \cap U=\{x\}$.

Let $x \in \mathcal{S}$ and let $U$ be a normal neighborhood of $x$. We define the local index $i(x, f)$ to be the local degree $\mu(f(x), f, U)$. For any other normal neighborhood $V$ of $x$ we have $\mu(f(x), f, V)=\mu(f(x), f, U)$ and thus $i(x, f)$ is well defined. It follows from basic properties of topological degree that for a sense-preserving branched cover $i(x, f)=\max _{y}$ card $f^{-1}(y) \cap U$, where $U$ is a normal neighborhood of $x$. For a Borel set $U$ we define $N(f, U)=\sup _{y} \operatorname{card} f^{-1}(y) \cap U$. In what follows, $U(x, r)$ stands for the $x$-component of $f^{-1} B(f(x), r)$.

Lemma 5.2. Assume $f: \mathcal{S} \rightarrow \mathbf{R}^{n}$ is a branched cover. For every $x \in \mathcal{S}$ there exists $s_{x}>0$ such that for $0<s<s_{x}$ the set $U(x, s)$ is a normal neighborhood of $x$ with $f U(x, s)=B(f(x), s)$. Furthermore

$$
\begin{aligned}
U(x, s) & =f^{-1} B(f(x), s) \cap U\left(x, s_{x}\right), \\
\partial U(x, s) & =f^{-1} \partial B(f(x), s) \cap U\left(x, s_{x}\right)
\end{aligned}
$$

and $\operatorname{diam} U(x, s) \rightarrow 0$ as $s \rightarrow 0$.

Proof. The proof is identical to the Euclidean case, see [16, Lemmata I.4.8 and II.4.1.]

Next we describe the path lifting property for sense-preserving branched covers. Let $\beta:\left[a, b\left[\rightarrow \mathbf{R}^{n}\right.\right.$ be a path. A maximal $f$-lifting of $\beta$ starting at point $x \in f^{-1}(\beta(a))$ is a path $\alpha:\left[a, c\left[\rightarrow \mathcal{S}, c \leq b\right.\right.$ such that $\alpha(a)=x, f \circ \alpha=\beta_{\mid[a, c[}$ and that if $c^{\prime}>c$ then there is no path $\alpha^{\prime}:\left[a, c^{\prime}\left[\rightarrow \mathcal{S}\right.\right.$ satisfying $f \circ \alpha^{\prime}=\beta_{\mid\left[a, c^{\prime}[\right.}$. 
Assume now that $x_{1}, x_{2}, \ldots, x_{k}$ are preimages of $\beta(a)$. Then there exists a maximal sequence $\alpha_{1}, \alpha_{2}, \ldots, \alpha_{m}$ of $f$-liftings of $\beta$ starting at points $x_{1}, x_{2}, \ldots, x_{k}$. This means that each $\alpha_{j}:\left[a, c_{j}\left[\rightarrow \mathcal{S}\right.\right.$ is a maximal $f$-lifting of $\beta$ starting at $x_{i}$ for some $i=1,2, \ldots k$ and

$$
m=\sum_{i=1}^{k} i\left(x_{i}, f\right) .
$$

Furthermore for every $i$

$$
\operatorname{card}\left\{j: \alpha_{j}(a)=x_{i}\right\}=i\left(x_{i}, f\right)
$$

and

$$
\operatorname{card}\left\{j: \alpha_{j}(s)=x\right\} \leq i(x, f),
$$

for every $s \in\left[a, c_{j}[\right.$ and $x \in \mathcal{S}$. The existence of the maximal sequence of liftings can be proved as in [16].

\section{Modulus inequalities for quasiregular maps}

In the Euclidean case the following lemma is known as $K_{0}$-inequality.

Lemma 6.1. Let $f: \mathcal{S} \rightarrow \mathbf{R}^{n}$ be a non-constant quasiregular map and let $\Gamma$ be a family of paths in a Borel set $U$ with $N(f, U)<\infty$. Then

$$
\operatorname{Mod}_{n} \Gamma \leq K_{0} N(f, U) \operatorname{Mod}_{n} f \Gamma .
$$

Proof. Let $\rho^{\prime} \in \operatorname{Adm} f \Gamma$. Define Borel function $\rho: \mathcal{S} \rightarrow \mathbf{R}$ by

$$
\rho(x)=\rho^{\prime}(f(x))\|D f(x)\|,
$$

for $x \in U$ and $\rho(x)=0$, for $x \in \mathcal{S} \backslash U$. Since $f \in N_{\text {loc }}^{1, n}\left(\mathcal{S}, \mathbf{R}^{n}\right)$, and $\|D f(x)\|$ is an $n$-weak upper gradient of $f$ it follows from [6, Proposition 5.3.4] that there exists $\Gamma_{0} \subset \Gamma$ with $\operatorname{Mod}_{n} \Gamma_{0}=\operatorname{Mod}_{n} \Gamma$ such that

$$
\int_{\gamma} \rho(s) d s=\int_{\gamma} \rho^{\prime}(f(s))\|D f(s)\| d s \geq \int_{f \circ \gamma} \rho^{\prime}(s) d s,
$$

for every $\gamma \in \Gamma_{0}$. Thus $\rho$ is admissible for $\Gamma_{0}$. Therefore by the distortion inequality and the coarea formula of Theorem 3.3, we get

$$
\begin{aligned}
\operatorname{Mod}_{n} \Gamma & =\operatorname{Mod}_{n} \Gamma_{0} \leq \int_{\mathcal{S}} \rho(x)^{n} d \mathcal{H}^{n}(x)=\int_{U} \rho^{\prime}(f(x))^{n}\|D f(x)\|^{n} d \mathcal{H}^{n}(x) \\
& \leq K_{O} \int_{U} \rho(f(x))^{n} J_{f}(x) d \mathcal{H}^{n}(x) \leq K_{O} \int_{U} \rho(y)^{n} N(f, U) d \mathcal{H}^{n}(y) .
\end{aligned}
$$

We do not have the opposite inequalities, such as Väisälä and Poletsky inequalities available. However, we have the following special case that suffices for our purposes. If $X=\mathbf{R}^{n}$ or $X=\mathcal{S}$ and $x \in X, r>0$ we write $\mathcal{S}^{n-1}(x, r)=\{y \in$ $X:|x-y|=r\}$.

Lemma 6.2. Let $f: \mathcal{S} \rightarrow \mathbf{R}^{n}$ be a non-constant quasiregular map and $x \in \mathcal{S}$. Let $s_{x}>0$ be as in Lemma 5.2 and $0<r<R<s_{x}$. Then

$$
m \omega_{n-1}\left(\log \frac{R}{r}\right)^{1-n} \leq K_{I} \operatorname{Mod}_{n} \Gamma\left(U_{r}, U_{R}^{c}\right) .
$$

Here we use notation $U(x, t)=U_{t}$ for every $t<s_{x}$ and $m=i(x, f)$. 
Proof. We may assume that $x=0$ and $f(x)=0$. For each $y \in \mathcal{S}^{n-1}(0,1) \subset$ $\mathbf{R}^{n}$ let $\gamma_{y}$ be the radial path $\gamma_{y}:[r, R] \rightarrow \mathbf{R}^{n}, \gamma_{y}(t)=t y$, connecting $\mathcal{S}^{n-1}(0, r)$ to $\mathcal{S}^{n-1}(0, R)$ and let $\Gamma$ be the family of paths $\gamma_{y}$. Then each $\gamma_{y} \in \Gamma$ has $m$ lifts $\alpha_{y, i}, i \in$ $\{1,2, \ldots, m\}$, lying on $U_{R}$ and each connecting $\bar{U}_{r}$ to $\overline{U_{R}^{c}}$. Let $\Gamma^{\prime}$ be the set of all such lifts. Let $\rho \in \operatorname{Adm} \Gamma\left(\bar{U}_{r}, \overline{U_{R}^{c}}\right)$. Then, in particular for each lift $\int_{\alpha_{y, i}} \rho \chi_{\bar{U}_{R} \backslash U_{r}} d s \geq 1$ and since each lift $\alpha_{y, i}$ is one to one, we have $\int_{\left|\alpha_{y, i}\right|} \rho(x) \chi_{\bar{U}_{R} \backslash U_{r}}(x) d \mathcal{H}^{1}(x) \geq 1$. Thus

$$
\omega_{n-1} m \leq \int_{\mathcal{S}^{n-1}(0,1)} \sum_{i=1}^{m} \int_{\left|\alpha_{y, i}\right|} \rho(x) \chi_{\bar{U}_{R} \backslash U_{r}}(x) d \mathcal{H}^{1}(x) d \mathcal{H}^{n-1}(y) .
$$

Since for each $x$ at most $i(x, f)$ of the lifts $\alpha_{y, i}$ pass through $x$, see Section 5 , we have

$$
\begin{aligned}
\omega_{n-1} m & \leq \int_{\mathcal{S}^{n-1}(0,1)} \int_{\bigcup_{i=1}^{m}\left|\alpha_{y, i}\right|} \rho(x) \chi_{\bar{U}_{R} \backslash U_{r}}(x) i(x, f) d \mathcal{H}^{1}(x) d \mathcal{H}^{n-1}(y) \\
& =\int_{\mathcal{S}^{n-1}(0,1)} \int_{\left(\frac{f}{|f|}\right)^{-1}(y)} \rho(x) \chi_{\bar{U}_{R} \backslash U_{r}}(x) i(x, f) d \mathcal{H}^{1}(x) d \mathcal{H}^{n-1}(y) .
\end{aligned}
$$

Here $\frac{f}{|f|}$ is a composition of a quasiregular map and a Lipschitz map. Hence the coarea formula is available and we get

$$
\omega_{n-1} m \leq \int_{\bar{U}_{R} \backslash U_{r}} \rho(x) i(x, f) J_{\frac{f}{|f|}}(x) d \mathcal{H}^{n}(x) \leq \int_{\bar{U}_{R} \backslash U_{r}} \rho(x) i(x, f) \frac{\left\|D^{\sharp} f(x)\right\|}{|f(x)|^{n-1}} d \mathcal{H}^{n}(x) .
$$

According to [10, Lemma 7.8], $i(x, f)=1$ whenever $f$ is differentiable at $x$ and $J_{f}(x)>0$. By this fact and the definition of $K_{I}$ it follows that

$$
\begin{aligned}
\omega_{n-1} m & \leq K_{I}^{\frac{1}{n}} \int_{\bar{U}_{R} \backslash U_{r}} \rho(x) \frac{J_{f}(x)^{\frac{n-1}{n}}}{|f(x)|^{n-1}} d \mathcal{H}^{n}(x) \\
& \leq K_{I}^{\frac{1}{n}}\left(\int_{\bar{U}_{R} \backslash U_{r}} \rho(x)^{n} d \mathcal{H}^{n}(x)\right)^{\frac{1}{n}}\left(\int_{\bar{U}_{R} \backslash U_{r}} \frac{J_{f}(x)}{|f(x)|^{n}} d \mathcal{H}^{n}(x)\right)^{\frac{n-1}{n}} .
\end{aligned}
$$

Here, again by the coarea formula

$$
\int_{\bar{U}_{R} \backslash U_{r}} \frac{J_{f}(x)}{|f(x)|^{n}} d \mathcal{H}^{n}(x) \leq \int_{B(0, R) \backslash B(0, r)} \frac{m}{|y|^{n}} d \mathcal{H}^{n}(y)=\omega_{n-1} m \log \frac{R}{r} .
$$

We conclude that

$$
\omega_{n-1} m\left(\log \frac{R}{r}\right)^{1-n} \leq K_{I} \int_{\bar{U}_{R} \backslash U_{r}} \rho(x)^{n} d \mathcal{H}^{n}(x) .
$$

This proves the lemma.

\section{A Bound for the linear inverse dilation and a modulus of continuity}

The concept of Loewner space turns out to be useful in our work.

Definition 7.1. We say that a metric measure space $X$ is an $n$-Loewner space, or satisfies the $n$-Loewner condition, if the function $\phi:(0, \infty) \rightarrow[0, \infty)$,

$$
\phi(t)=\inf \left\{\operatorname{Mod}_{n} \Gamma(E, F): \triangle(E, F) \leq t\right\}
$$

is positive whenever $E, F$ are two nondegenerate disjoint continua in $X$. Here

$$
\triangle(E, F)=\frac{\operatorname{dist}(E, F)}{\min \{\operatorname{diam} E, \operatorname{diam} F\}} .
$$


By [3, Proposition 4.4] a proper path connected metric measure space supporting a weak $n$-Poincaré inequality is quasiconvex. Thus [5, Theorem 5.7] allows us to conclude that a space $\mathcal{S}$ satisfying the axioms listed in Section 2 is an $n$-Loewner space.

Definition 7.2. The linear dilation $H(x, f)$ and inverse linear dilation $H^{*}(x, f)$ are defined as follows.

where

$$
H(x, f)=\limsup _{r \rightarrow 0} \frac{L(x, r)}{l(x, r)}
$$

$$
\begin{aligned}
L(x, r) & =\max \{|f(x)-f(y)|:|x-y|=r\}, \\
l(x, r) & =\min \{|f(x)-f(y)|:|x-y|=r\}
\end{aligned}
$$

and

where

$$
H^{*}(x, f)=\limsup _{r \rightarrow 0} \frac{L^{*}(x, r)}{l^{*}(x, r)}
$$

$$
\begin{aligned}
l^{*}(x, r) & =\min _{y \in \partial U(x, r)}|x-y|, \\
L^{*}(x, r) & =\max _{y \in \partial U(x, r)}|x-y| .
\end{aligned}
$$

Recall that $U(x, r)$ is the $x$-component of $f^{-1} B(f(x), r)$.

Theorem 7.3. Let $f: \mathcal{S} \rightarrow \mathbf{R}^{n}$ be a nonconstant quasiregular map. Let $x \in \mathcal{S}$ and let $s_{x}>0$ be as in Lemma 5.2. There exists a constant $H^{*}$ depending only on $K_{O}$ and $\mathcal{S}$, such that for every $0<r<s_{x}$ with $L^{*}(x, r)<l^{*}\left(x, s_{x}\right)$, we have

$$
\frac{L^{*}(x, r)}{l^{*}(x, r)} \leq H^{*}
$$

Remark 7.4. Note that by Lemma 5.2 for small $r>0$ the condition $L^{*}(x, r)<$ $l^{*}\left(x, s_{x}\right)$, is always satisfied.

We start with the following lemma which gives lower bounds for modulus needed in the proof of Theorem 7.3.

Lemma 7.5. Fix $x \in \mathcal{S}$ and $s_{x}$ as in Lemma 5.2. If $t \leq s_{x}$ we write $U_{t}$ for $U(x, t)$. Fix $0<r<s_{x}$. Denote $l_{r}^{*}=l^{*}(x, r), L_{r}^{*}=L^{*}(x, r)$ and let $l=l\left(x, l_{r}^{*}\right)$, $L=L\left(x, L_{r}^{*}\right)$. Assume that $L<s_{x}$. Then there exists a constant $C>0$ depending only on $\mathcal{S}$ such that

$$
C \leq \min \left\{\operatorname{Mod}_{n} \Gamma\left(U_{l}, U_{r}^{c}\right), \operatorname{Mod}_{n} \Gamma\left(U_{r}, U_{L}^{c}\right)\right\} .
$$

Proof. Since $\mathcal{S}$ is an $n$-Loewner space, in order to find a lower bound for $\operatorname{Mod}_{n} \Gamma\left(U_{l}, U_{r}^{c}\right)$ it suffices to find an upper bound for

$$
\frac{\operatorname{dist}\left(U_{l}, U_{r}^{c}\right)}{\min \left\{\operatorname{diam} U_{l}, \operatorname{diam} U_{r}^{c}\right\}} \text {. }
$$

To see that $\min \left\{\operatorname{diam} U_{l}, \operatorname{diam} U_{r}^{c}\right\}=\operatorname{diam} U_{l} \geq l_{r}^{*}$, let $z \in \mathcal{S}^{n-1}\left(x, l_{r}^{*}\right)$ such that

$$
|f(z)-f(x)|=\min _{z^{\prime} \in \mathcal{S}^{n-1}\left(x, l_{r}^{*}\right)}\left|f\left(z^{\prime}\right)-f(x)\right| .
$$

Then

$$
f(z) \in \partial B(f(x), l)
$$

but by Lemma $5.2 f^{-1} \partial B(f(x), l) \cap U_{s_{x}}=\partial U_{l}$ and thus $z \in \partial U_{l}$. Therefore $\overline{U_{l}}$ is a connected set containing $x$ and $z \in \mathcal{S}^{n-1}\left(x, l_{r}^{*}\right)$ and so diam $U_{l} \geq l_{r}^{*}$. Since there 
exists $y \in \partial U_{r}$ such that $|y-x|=l_{r}^{*}$ and because $x \in U_{l}$ we see that $\operatorname{dist}\left(U_{r}^{c}, U_{l}\right) \leq l_{r}^{*}$. Thus

$$
\frac{\operatorname{dist}\left(U_{l}, U_{r}^{c}\right)}{\min \left\{\operatorname{diam} U_{l}, \operatorname{diam} U_{r}^{c}\right\}} \leq \frac{l_{r}^{*}}{l_{r}^{*}}=1 .
$$

Next consider $\operatorname{Mod}_{n} \Gamma\left(U_{r}, U_{L}^{c}\right)$. For the lower bound of the modulus it suffices to find an upper bound for

$$
\frac{\operatorname{dist}\left(U_{r}, U_{L}^{c}\right)}{\min \left\{\operatorname{diam} U_{r}, \operatorname{diam} U_{L}^{c}\right\}} .
$$

It is an immediate consequence of the definition of $L_{r}^{*}=L^{*}(x, r)$ that $\operatorname{diam} U_{r} \geq L_{r}^{*}$. To see that $\partial U_{L} \cap \mathcal{S}^{n-1}\left(x, L_{r}^{*}\right) \neq \emptyset$, let $z \in \mathcal{S}^{n-1}\left(x, L_{r}^{*}\right)$ such that $|f(x)-f(z)|=$ $L\left(x, L_{r}^{*}\right)=L$. Then

$$
f(z) \in \partial B(f(x), L)
$$

and thus again Lemma 5.2 and the assumption $L<s_{x}$ give $z \in \partial U_{L}$. Therefore $\partial U_{L} \cap \mathcal{S}\left(x, L_{r}^{*}\right) \neq \emptyset$. Since $x \in U_{r}$ we see that $\operatorname{dist}\left(U_{r}, U_{L}^{c}\right) \leq L_{r}^{*}$. Hence

$$
\frac{\operatorname{dist}\left(U_{r}, U_{L}^{c}\right)}{\min \left\{\operatorname{diam} U_{r}, \operatorname{diam} U_{L}^{c}\right\}} \leq \frac{L_{r}^{*}}{L_{r}^{*}}=1 .
$$

This proves the lemma.

Now we are ready to prove Theorem 7.3.

Proof of Theorem 7.3. We will use the notation of Lemma 7.5 and denote $m=i(x, f)$. By Lemmata 7.5 and 6.1 , we have

$$
C(\mathcal{S}) \leq \operatorname{Mod}_{n} \Gamma\left(U_{l}, U_{r}^{c}\right) \leq K_{0} m \operatorname{Mod}_{n} f \Gamma\left(U_{l}, U_{r}^{c}\right)
$$

and

$$
C(\mathcal{S}) \leq \operatorname{Mod}_{n} \Gamma\left(U_{r}, U_{L}^{c}\right) \leq K_{0} m \operatorname{Mod}_{n} f \Gamma\left(U_{r}, U_{L}^{c}\right)
$$

Note that every path in $f \Gamma\left(U_{l}, U_{r}^{c}\right)$ has a subpath connecting $\mathcal{S}^{n-1}(f(x), l)$ to $\mathcal{S}^{n-1}(f$ $(x), r)$ and thus $\operatorname{Mod}_{n} f \Gamma\left(U_{l}, U_{r}^{c}\right) \leq \omega_{n-1}\left(\log \frac{r}{l}\right)^{1-n}$, see [19, Theorem 6.4 and Example 7.5]. Similarly, every path in $f \Gamma\left(U_{r}, U_{L}^{c}\right)$ has a subpath connecting $\mathcal{S}^{n-1}(f(x), r)$ to $\mathcal{S}^{n-1}(f(x), L)$ and thus $\operatorname{Mod}_{n} f \Gamma\left(U_{r}, U_{L}^{c}\right) \leq \omega_{n-1}\left(\log \frac{L}{r}\right)^{1-n}$. This gives

$$
\max \left\{\left(\log \frac{r}{l}\right)^{n-1},\left(\log \frac{L}{r}\right)^{n-1}\right\} \leq \frac{\omega_{n-1} K_{O} m}{C(\mathcal{S})} .
$$

By Lemma 6.2

$$
m \omega_{n-1}\left(\log \frac{L}{l}\right)^{1-n} \leq K_{I} \operatorname{Mod}_{n} \Gamma\left(U_{l}, U_{L}^{c}\right)
$$

On the other hand, every $\gamma \in \Gamma\left(U_{l}, U_{L}^{c}\right)$ has a subpath connecting $B\left(x, l_{r}^{*}\right)$ to $B\left(x, L_{r}^{*}\right)$ and thus $\operatorname{Mod}_{n} \Gamma\left(U_{l}, U_{L}^{c}\right) \leq \operatorname{Mod}_{n} \Gamma\left(B\left(x, l_{r}^{*}\right), B\left(x, L_{r}^{*}\right)\right)$. It holds even in a greater generality that

$$
\operatorname{Mod}_{n} \Gamma\left(B(x, s), B(x, R)^{c}\right) \leq C_{0}\left(\log \frac{R}{s}\right)^{1-n},
$$

see [6, Proposition 4.3.10]. Here $C_{0}$ depends only on the Ahlfors regularity constant $C_{A}$ of Definition 2.5. Hence

$$
m \omega_{n-1}\left(\log \frac{L}{l}\right)^{1-n} \leq K_{I} \operatorname{Mod}_{n} \Gamma\left(U_{l}, U_{L}^{c}\right) \leq K_{I} C_{0}\left(\log \frac{L_{r}^{*}}{l_{r}^{*}}\right)^{1-n} .
$$

By (16) and (17) we conclude

$$
\frac{m \omega_{n-1}}{K_{I} C_{0}}\left(\log \frac{L_{r}^{*}}{l_{r}^{*}}\right)^{n-1} \leq\left(\log \frac{L}{l}\right)^{n-1} \leq\left(\log \frac{L}{r}+\log \frac{r}{l}\right)^{n-1} \leq 2^{n-1} \frac{\omega_{n-1} K_{0} m}{C(\mathcal{S})}
$$


This is

which proves the claim.

$$
\left(\log \frac{L_{r}^{*}}{l_{r}^{*}}\right)^{n-1} \leq 2^{n-1} \frac{C_{0} K_{I} K_{O}}{C(\mathcal{S})}
$$

Applying the results above we are able to prove the following modulus of continuity.

Theorem 7.6. Let $f: \mathcal{S} \rightarrow \mathbf{R}^{n}$ be a non-constant quasiregular map and $x \in \mathcal{S}$. There exist $A, \rho>0$ depending on $x$ such that for every $y \in B(x, \rho)$

$$
|f(x)-f(y)| \leq A|x-y|^{\mu},
$$

where $\mu=\left(\frac{\omega_{n-1} i(x, f)}{C_{0} K_{I}}\right)^{\frac{1}{n-1}}$. Here $C_{0}$ depends only on Ahlfors regularity constant $C_{A}$.

Proof. Let $s_{x}$ be as in Lemma 5.2 and $r_{0}$ so small that $L^{*}\left(x, r_{0}\right)<l^{*}\left(x, s_{x}\right)$. Set $\rho_{1}=l^{*}\left(x, s_{x}\right)$ and $\rho=l^{*}\left(x, r_{0}\right)$. Fix $y \in B(x, \rho)$ and let $r=|f(x)-f(y)|$. Then $r<s_{x}$, and Lemma 6.2 gives

$$
m \omega_{n-1}\left(\log \frac{s_{x}}{r}\right)^{1-n} \leq K_{I} \operatorname{Mod}_{n} \Gamma\left(U(x, r), U\left(x, s_{x}\right)^{c}\right)
$$

On the other hand, every path in $\Gamma\left(U(x, r), U\left(x, s_{x}\right)^{c}\right)$ has a subpath connecting $B\left(x, L_{r}^{*}\right)$ to $B\left(x, \rho_{1}\right)^{c}$, here $L_{r}^{*}=L^{*}(x, r)$. Thus, as in the proof of Theorem 7.3 , we get

This together with (18) gives

$$
\operatorname{Mod}_{n} \Gamma\left(U(x, r), U\left(x, s_{x}\right)^{c}\right) \leq C_{0}\left(\log \frac{\rho_{1}}{L_{r}^{*}}\right)^{1-n} .
$$

$$
m \omega_{n-1}\left(\log \frac{s_{x}}{r}\right)^{1-n} \leq K_{I} C_{0}\left(\log \frac{\rho_{1}}{L_{r}^{*}}\right)^{1-n} .
$$

Hence

$$
r \leq s_{x} \rho_{1}^{-\mu}\left(L_{r}^{*}\right)^{\mu}
$$

According to Theorem 7.3 there exists a constant $H^{*}\left(\mathcal{S}, K_{O}\right)$ such that $L_{r}^{*} \leq H^{*} l^{*}(x, r)$. On the other hand, $l^{*}(x, r) \leq|x-y|$ and thus we obtain

$$
|f(x)-f(y)| \leq s_{x} \rho_{1}^{-\mu} H^{* \mu}|x-y|^{\mu} .
$$

This proves the Theorem with $A=s_{x} l^{*}\left(x, s_{x}\right)^{-\mu} H^{* \mu}$.

Remark 7.7. Note that since $H^{*}$ does not depend on the local index $i(x, f)$ the theorem above gives a better modulus of continuity around those points whose local index is large.

Acknowledgements. The author wishes to thank Professor Kai Rajala for helpful discussions. Part of this research was done when the author was visiting at the University of Michigan. He wishes to thank the department for its hospitality.

\section{References}

[1] Bojarski, B., and T. Iwaniec: Analytical foundations of the theory of quasiconformal mappings in $\mathbf{R}^{n}$. - Ann. Acad. Sci. Fenn. Ser. A I Math. 8:2, 1983, 257-324.

[2] Federer, H.: Geometric measure theory. - Grundlehren Math. Wiss. 153, Springer-Verlag, New York, 1969.

[3] HajŁasz, P., and P. Koskela: Sobolev met Poincaré. - Mem. Amer. Math. Soc. 145:688, 2000. 
[4] Heinonen, J., and S. KeIth: Flat forms, bi-Lipschitz parameterizations, and smoothability of manifolds. - Publ. Math. Inst. Hautes Études Sci. 113, 2011, 1-37.

[5] Heinonen, J., and P. Koskela: Quasiconformal maps in metric spaces with controlled geometry. - Acta Math. 181:1, 1998, 1-61.

[6] Heinonen, J., P. Koskela, N. Shanmugalingam, and J. Tyson: Sobolev Spaces on metric measure spaces. An approach based on upper gradients. - New Math. Monogr. 27. Cambridge Univ. Press, Cambridge, 2015.

[7] Heinonen, J., and S. Rickman: Geometric branched covers between generalized manifolds. - Duke Math. J. 113:3 2002, 465-529.

[8] Heinonen, J., and D. Sullivan: On the locally branched Euclidean metric gauge. - Duke Math. J. 114:1, 2002, 15-41.

[9] Iwaniec, T., and G. Martin: Geometric function theory and non-linear analysis. - Oxford Math. Monogr., The Clarendon Press, Oxford Univ. Press, New York, 2001.

[10] Kirsilë, V.: Mappings of finite distortion from generalized manifolds. - Conform. Geom. Dyn. $18,2014,229-262$.

[11] Lindqvist, P.: On the quasiregularity of a limit mapping. - Ann. Acad. Sci. Fenn. Ser. A I Math. 11:2, 1986, 155-159.

[12] Martio, O.: A capacity inequality for quasiregular mappings. - Ann. Acad. Sci. Fenn. Ser. A I Math. 474, 1970, 1-18.

[13] Mattila, P.: Geometry of sets and measures in Euclidean spaces. - Cambridge Univ. Press, Cambridge, 1995.

[14] RešEtnjak, Ju. G.: Mappings with bounded distortion as extremals of integrals of Dirichlet type. - Sibirsk. Mat. Ž. 9, 1968, 652-666 (in Russian).

[15] ReŠEtnjak, Ju. G.: Space mappings with bounded distortion. - Transl. Math. Monogr. 73, Amer. Math. Soc., Providence, RI, 1986.

[16] Rickman, S.: Quasiregular mappings. - Springer-Verlag, Berlin, 1993.

[17] Semmes, S.: Finding curves on general spaces through quantitative topology, with applications to Sobolev and Poincaré inequalities. - Selecta Math. (N.S.) 2, 1996, 155-295.

[18] Shanmugalingam, N.: Newtonian spaces: an extension of Sobolev spaces to metric measure spaces. - Rev. Mat. Iberoamericana 16:2, 2000, 243-279.

[19] VÄIsÄLÄ, J.: Lectures on $n$-dimensional quasiconformal mappings. - Lecture Notes in Math. 229, Springer-Verlag, Berlin-New York, 1971.

[20] Zatorska-Goldstein, A.: Very weak solutions of nonlinear subelliptic equations. - Ann. Acad. Sci. Fenn. Math. 30:2, 2005, 407-436. 